\title{
A Study of Self-Propelled Elastic Cylindrical Micro-swimmers using Modeling and Computation
}

\author{
Lingling Shi ${ }^{a}$, Sunčica Čanić* ${ }^{* a}$, Annalisa Quaini ${ }^{a}$, Tsorng-Whay Pan ${ }^{\text {a }}$ \\ ${ }^{a}$ Department of Mathematics, University of Houston, 4800 Calhoun Rd, Houston TX 77204, USA
}

\begin{abstract}
We study propulsion of micro-swimmers in 3D creeping flow. The swimmers are assumed to be made of elastic cylindrical hollow tubes. The swimming is generated by the contractions of the tube's elastic membrane walls producing a traveling wave in the form of a "step-function" traversing the swimmer from right to left, propelling the swimmer from left to right. The problem is motivated by medical applications such as drug delivery. The influence of several non-dimensional design parameters on the velocity of the swimmer is investigated, including the swimmer aspect ratio, and the amplitude of the traveling wave relative to the swimmer radius. An immersed boundary method based on a finite element method approach is successfully combined with an elastic spring network model to simulate the two-way fluid-structure interaction coupling between the elastic cylindrical tube and the flow of a 3D viscous, incompressible fluid. To gain a deeper insight into the influence of various parameters on the swimmer speed, a reduced 1D fluid-structure interaction model was derived and validated. It was found that fast swimmers are those with large tube aspect ratios, and with the amplitude of the traveling wave which is roughly $50 \%$ of the reference swimmer radius. It was shown that the speed of our "optimal swimmer" is around 1.5 swimmer lengths per second, which is at the top of the class of all currently manufactured micro-swimmers swimming in low Reynolds number flows $\left(R e=10^{-6}\right)$, reported in [11].
\end{abstract}

Keywords Micro-swimmers, Immersed boundary method, Fluid-structure interaction, Reduced 1D model.

\section{Introduction}

Micro robots, or micro-swimmers, are a class of artificial nano- or micro-scale particles capable of converting external energy into motion. They swim in low Reynolds number regimes $\left(R e=10^{-6}\right)$ where viscosity dominates. Due to the reversibility in low Reynolds number flow (e.g. Stokes flow) the action of swimming micro organisms in nature are different from regular size swimmers such as fish [30]. New propulsion methodologies are needed to efficiently move these swimmers. It turns out that all the micro-swimming organisms create in one way or another a traveling wave, moving in the opposite direction of the swimmer motion (see e.g., spermatozoa [5, 24], celia [19], amoeba [3], and anguilliform swimmers [14, 28]). Such a traveling wave can be artificially generated in synthetic swimmers by using, e.g., piezoelectric [16], ICFP [13], or magnetic actuators [9, 31, 17]. Applications of such micro-swimmers include biomedical applications such as drug delivery ("smart pills" [10]) and diagnostic tools. Biological low Reynolds number flow regimes can be found in, for example, blood flow in arterioles and in capillary beds, which populate all of our organs, in mammalian productive systems [4], and in intestines [26]. A comprehensive review of the literature on manufactured mili and micro medical swimmers can be found in [11].

\footnotetext{
* Corresponding author

Email addresses: shi@math.uh.edu (Lingling Shi), canic@math.uh.edu (Sunčica Čanić), quaini@math.uh.edu (Annalisa Quaini), pan@math.uh.edu (Tsorng-Whay Pan)
} 


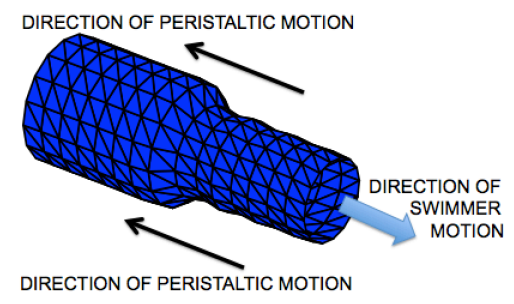

Figure 1: A sketch of cylindrical swimmer during peristaltic motion.

In this manuscript we study performance of synthetic micro-swimmers, manufactured from a hollow, elastic cylindrical tube. See Figure 1. The tube wall is an elastic membrane generating a peristaltic traveling wave produced by the contractions of the cylindrical membrane. The contractions are driven by an outside energy source such as an actuator, or, in the future, by the contractions of cardiac cells called myocytes $[18,15]$. The traveling wave moving from right to left generates displacements of mass to exchange momentum between the swimming body and the surrounding fluid, causing the swimmer to move in the opposite direction of the traveling wave motion. We study the influence of various swimmer design parameters, such as the aspect ratio of the swimmer, and the amplitude of the peristaltic wave motion, on the speed generated by the swimmer. Mathematical modeling, analysis, and computer simulations are used to perform this study.

The mathematical approach is based on 3D fluid-structure interaction (FSI) simulations modeling the interaction between an elastic swimmer membrane (i.e., structure) and the surrounding fluid. The swimmer is modeled as a nonlinearly elastic cylindrical membrane/shell by using a 3D elastic spring network model $[29,25,27]$, while the fluid flow is modeled by using the Navier-Stokes equations for a viscous, incompressible fluid. Two coupling conditions are employed to model the interaction between the fluid and structure: kinematic condition describing continuity of velocities, i.e., the no-slip condition, and dynamic condition describing balance of contact forces holding at the swimmer surface. The FSI problem is solved using the Immersed Boundary Method [21, 22, 23], combined with a time-discretization via Lie operator splitting [12]. At every time step the Lie splitting separates the fluid from structure sub-problems. We used a Fictitious Domain Method with Lagrange Multipliers [12] to solve the fluid subproblem. Regarding spatial discretization, a Finite Element Method approach was used to discretized the fluid sub-problem, while the elastic swimmer membrane is discretized using a set of triangular elements whose vertices are connected by elastic springs. The total energy of the membrane accounts for the energy due to the in-plane change of metric (stretching/compression), and the energy due to the outof-plane bending effects (change in curvature). The contractions of the membrane are modeled by an external force acting on the membrane surface in the normal direction. This approach, which combines the Immersed Boundary Method with Lie splitting and the Fictitious Domain Method with Lagrange Multipliers was introduced recently in [25] to study fluid-structure interaction with deformable red blood cells.

The resulting 3D model is used to investigate how the velocity of the synthetic swimmer depends on the aspect ratio of the swimmer cylinder (reference radius versus reference length), and on the amplitude of the generated traveling wave (i.e., the ratio between the squeezed radius versus the reference radius). Interesting results were obtained.

Firstly, during one cycle of application of force causing one traveling wave traversing the cylinder from right to left, the swimmers that moved the farthest are those that displaced the largest amount of fluid during one cycle, as expected. This means, in particular, that for a fixed reference radius of the swimmer and a given amplitude of the traveling wave, the longer the swimmer the farther it moves. Similarly, for a fixed reference radius and length of the swimmer, the larger the amplitude of the traveling 
wave, the longer the distance traversed by the swimmer. The time interval corresponding to one cycle of force application varied by the swimmer.

However, when multiple cycles of force application were considered during a fixed time interval, different results were obtained. In terms of the aspect ratio, short swimmers, i.e., the swimmers with large aspect ratios, moved the farthest within a given time interval. We found that such swimmers have the largest average velocity per one cycle, as they produce the largest rate of change of displaced mass per unit time.

In terms of the traveling wave amplitude, the swimmers experiencing roughly $50 \%$ of radius displacement moved the farthest within a given time interval. We found, again, that such swimmers have the largest average velocity in one cycle.

Thus, fast swimmers are those with large aspect ratios whose radius deforms roughly $50 \%$ in each cycle. Such swimmers are capable of generating a large number of contractions per unit time because of their short length, producing the largest rate of change of displaced mass per unit time.

To get a better insight into the influence of different parameters on the velocity of the swimmers, we developed a nonlinear 1D reduced model of fluid-structure interaction between a slender (linear) swimmer and the flow of a creeping fluid. A similar 1D model was also considered in [2] for a spherical swimmer with internally generated traveling waves. We show that the reduced model approximates well our 3D FSI simulations of slender swimmers that deform up to $50 \%$ of the reference radius.

Finally, we show that our fastest swimmers travel 1.5 swimmer lengths per second, which is near the top of the class of all currently available manufactured artificial swimmers swimming at low Reynolds numbers. More precisely, in a recent review article [11] it was reported that at Reynolds numbers of $10^{-6}$, which is our flow regime, the currently manufactured artificial swimmers typically swim at the speed between 0.6 swimmer lengths per second $(15-\mathrm{Au} / \mathrm{Ni} / \mathrm{Au} / \mathrm{Pt}-\mathrm{CNT}$ motor with $4.3 \mu m$ diameter cargo), and 2 swimmer lengths per second (striped metallic nano rod). Thus, our results appear to be quite relevant, and are a first step in our long-term goal to produce cylindrical medical micro-swimmers driven by the contractions of cardiac myocytes [15, 18].

\section{Problem description, mathematical model, and numerical solver}

\subsection{Problem Description}

We study propulsion of an elastic cylindrical (hollow) tube by a peristaltic motion of its elastic membrane walls, moving through a viscous, incompressible fluid at low Reynolds numbers. The elastic cylindrical membrane is immersed in the fluid occupying a larger cylinder with rigid walls, whose radius is much larger than that of the swimmer so that it does not influence the swimmer motion. The reference configuration of the swimmer is a straight cylinder of radius $R_{0}$ and length $L$. A traveling wave moving from right to left is induced in the cylinder membrane, see Figure 1, producing an overall motion of the cylinder in the opposite direction from the traveling wave. The traveling wave is generated by the application of force to the surface of the cylinder wall in the radial direction by employing the following strategy. The swimmer surface is divided into $N$ segments $\left(\left(0, l_{1}\right),\left(l_{1}, l_{2}\right), \cdots,\left(l_{N-1}, L_{N}\right)\right)$, and a force sequence $F_{i}$ is applied to the segments $\left(l_{i-1}, l_{i}\right)$ for the time duration of $t_{i}$ seconds, $i=N, N-1, \cdots, 1$, see Figure 2 (left), traversing the swimmer surface from right to left to produce a traveling wave moving from right to left. We will call this wave a peristaltic wave.

The goal of this work is to investigate the influence of the following non-dimensional parameters on the motion, i.e., speed of the swimmer:

1. The ratio between the swimmer radius $R_{0}$ and length $L$ :

$$
\epsilon:=R_{0} / L
$$



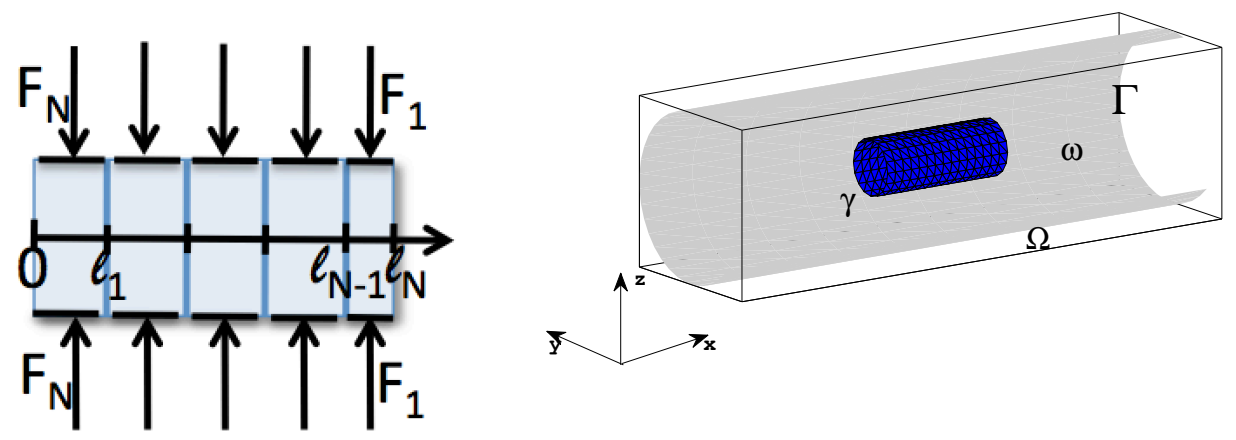

Figure 2: Left: A sketch of force application strategy. Right: Computational domain with elastic swimmer.

2. The ratio between the swimmer deformed radius $R_{f}$ and the reference radius $R_{0}$ :

$$
\lambda:=R_{f} / R_{0}
$$

i.e., the relative amplitude of the peristaltic wave.

Related to the velocity is the distance traveled by the swimmer. We will investigate the distance traveled by the swimmer during:

- One peristaltic contraction of the swimmer, i.e., one cycle of force application (the time interval is not fixed; it depends on the swimmer); and

- Multiple peristaltic contractions of the swimmer (during a fixed time interval).

The results are not as straight-forward as one might expect. They are influenced by the amount of displaced mass, the rate of change of displaced mass, the nonlinearity of the swimmer model, the fluid viscosity, and by the "added mass effect" associated with the displaced fluid during swimmer contractions.

We remark here that one way to introduce a peristaltic motion of the swimmer would have been to impose the location of the swimmer membrane a priori, prescribing the peristaltic wave independently of the fluid surrounding the swimmer. This would have been a much easier task to study from both the mathematical and computational points of view, since this approach defines a fluid-structure interaction problem with one-way coupling due to the prescribed structure motion. In practice, however, peristaltic motion of the swimmer is produced by the action of a certain force on the swimmer surface, which gives rise to a fluid-structure interaction problem with two-way coupling between the fluid and structure. It turns out that to produce a peristaltic way for such a swimmer immersed in the fluid is a non-trivial task, since the swimmer deformation and its motion depend nonlinearly not only on the elastic properties of the swimmer membrane, but also on the fluid stress exerted by the fluid onto the swimmer. Our results, presented below, suggest a practical strategy of application of force onto the swimmer surface to produce a desired peristaltic swimmer motion, which may be adopted in the design of an actual swimmer.

\subsection{Mathematical Model}

The mathematical problem is that of fluid-structure interaction between an incompressible, viscous fluid, and elastic cylinder immersed in the fluid. The numerical approach to solve this fluid-structure interaction problem in this work is based on the Immersed Boundary Method coupled with the Fictitious Domain Method with Lagrange Multipliers to solve the corresponding fluid sub-problem. The general 
mathematical framework of the problem is given in the context of the Immersed Boundary Method formulation (IBM).

We begin by defining the fluid and structure models, and then define the coupled fluid-structure interaction problem in the context of IBM.

The fluid model. The fluid motion will be modeled by the time-dependent Stokes (Navier-Stokes) equations for an incompressible, viscous fluid defined inside a large cylindrical "fluid container", which we denote by $\omega$. See Figure 2 (right). Since our 3D methodology works for the full Navier-Stokes equations, we present the model problem for the Navier-Stokes equations and then indicate how simplifications of the problem can be done to solve the time-dependent Stokes problem. The Navier-Stokes equations, defined on a time interval $(0, T), T>0$, read as follows:

$$
\begin{aligned}
& \rho\left(\frac{\partial \mathbf{u}}{\partial t}+\mathbf{u} \cdot \nabla \mathbf{u}\right)=\nabla \boldsymbol{\sigma}+\mathbf{f} \text { in } \omega \times(0, T), \\
& \nabla \cdot \mathbf{u}=0 \text { in } \omega \times(0, T) .
\end{aligned}
$$

Here $\mathbf{u}$ is the fluid velocity, $\rho$ is the fluid density, $\mathbf{f}$ is the body force, and $\boldsymbol{\sigma}=-p \mathbf{I}+2 \mu \mathbf{D}(\mathbf{u})$ is the fluid Cauchy stress tensor for Newtonian fluids, where $p$ is the fluid pressure, $\mu$ is the kinematic viscosity coefficient, and $\mathbf{D}(\mathbf{u})=\frac{1}{2}\left(\nabla \mathbf{u}+\nabla^{\tau} \mathbf{u}\right)$ is the symmetrized gradient of $\mathbf{u}$.

For low Reynolds number flows the convection term $\mathbf{u} \cdot \nabla \mathbf{u}$ is small and can be neglected, giving rise to the time-dependent (linear) Stokes equations. This will be the case with our swimmer.

Equations (1) and (2) are supplemented with the no-slip boundary condition on the boundary $\Gamma$ of $\omega$, and an initial condition $\mathbf{u}_{0}$ for the fluid velocity, which we normally take to be equal to zero:

$$
\begin{aligned}
& \mathbf{u}=\mathbf{0} \text { on } \Gamma \times(0, T), \\
& \mathbf{u}(\mathbf{x}, 0)=\mathbf{u}_{0}(\mathbf{x}) \text { in } \omega .
\end{aligned}
$$

The elastic swimmer model. We will be assuming that our elastic swimmer behaves as a (nonlinearly) elastic membrane/shell. The model that we present below will be of the form

$$
m \ddot{\mathbf{r}}-\mathbf{F}(\mathbf{r})=\mathbf{F}^{e x t},
$$

where $m$ is the mass density of the swimmer, $\mathbf{r}$ is a vector describing the location of points of the swimmer, $\mathbf{F}$ is the force due to the elastic and bending energy of the swimmer, $\mathbf{F}^{\text {ext }}$ is the external force density, and 'denotes the time derivative. The term $\mathbf{F}(\mathbf{r})$ will be obtained as the derivative of the energy density with respect to $\mathbf{r}$, where the energy density will account for the energy due to the in-plane change of metric due to stretching/compression (membrane effects), and the energy associated with the change in curvature due to the out-of-plane bending effects (shell effects). These ideas are incorporated
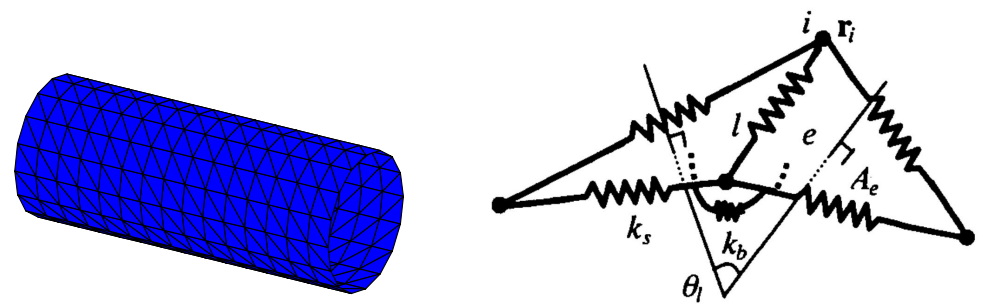

Figure 3: Swimmer membrane discretization using elastic springs.

into a three-dimensional elastic spring network, developed and used in [29, 25, 27]. Based on this model, the (middle surface of the) swimmer membrane/shell can be viewed as a collection of small triangular elements whose vertices are connected by springs, as shown in Figure 3. The total number of vertices 
(nodes) will be denoted by $N_{n}$, while the total number of springs by $N_{s}$. The elastic energy of the swimmer membrane/shell includes the energy stored due to the change of the length $L_{l}$ of each spring with respect to its reference length $L_{l}^{0}$, and the energy stored due to the change in the angle $\theta_{l}$ between two neighboring triangular elements. Thus, the elastic energy of the swimmer membrane

$$
E=E_{e}+E_{b}
$$

is the sum of the total energy due to stretch/compression

$$
E_{e}=\frac{k_{s}}{2} \sum_{l=1}^{N_{s}}\left(L_{l}-L_{l}^{0}\right)^{2},
$$

and the total energy due to bending

$$
E_{b}=\frac{k_{b}}{2} \sum_{l=1}^{N_{s}} L_{l} \tan ^{2}\left(\frac{\theta_{l}}{2}\right) .
$$

Here $k_{s}$ and $k_{b}$ are the spring constants accounting for the changes in length and bending angle, respectively, and $\theta_{l}$ is the angle between the normal outer vectors of the two neighboring triangular elements, which have the side $l$ as their boundary. In equation (8) the tangent function was chosen to avoid the folding of springs at large bending deformations.

We note that in $[29,25,27]$ additional terms were considered in the definition of the elastic energy of the membrane to enforce the local and global area conservation. This was done because in those works the focus was on modeling the elastic properties of red blood cells. These additional terms are related to the incompressibility of the membrane material, and to the total volume conservation within a cell, which we do not have in our problem. In our model we dropped the area conservation property to be able to capture, more realistically, the traveling wave in our swimmer membrane/shell.

To describe the elastodynamics of the swimmer we assign to each node (apex) $i$ of the triangular elements the position vector $\mathbf{r}_{i}$. Using vector analysis, the right hand-side of the energy function (6) can be written as a function of $\mathbf{r}_{i}$. Based on the principle of virtual work, the force acting on the $i$ th membrane node due to the elastic energy is then given by

$$
\mathbf{F}_{i}=-\frac{\partial E}{\partial \mathbf{r}_{i}},
$$

where $\mathbf{F}_{i}=\mathbf{F}_{i}\left(\mathbf{r}_{1}, \ldots, \mathbf{r}_{N_{n}}\right)$. We will be assuming that the swimmer membrane has a certain mass, so that each node $i$ is assigned mass $m$ such that the total mass is equal to $m N_{n}$. If we assume that external force $\mathbf{F}^{e x t}$ is applied to the swimmer so that at each node the external force equals $\mathbf{F}_{i}^{e x t}$, then each swimmer node moves based on the following equation of motion:

$$
m \ddot{\mathbf{r}}_{i}-\mathbf{F}_{i}=\mathbf{F}_{i}^{e x t} .
$$

External force $\mathbf{F}_{i}^{e x t}$ in this FSI problem will include the force exerted by the fluid onto the structure, i.e., the force that the structure feels from the fluid, given in Lagrangian coordinates by $\overline{\mathbf{F}}_{i}$, and the force $\mathbf{F}_{i}^{e}$ exerted by other external sources such as, e.g., the actuators, causing the swimmer membrane to contract to produce a traveling wave responsible for the peristaltic motion of the swimmer. Thus,

$$
m \ddot{\mathbf{r}}_{i}-\mathbf{F}_{i}=\overline{\mathbf{F}}_{i}+\mathbf{F}_{i}^{e} .
$$

The position $\mathbf{r}_{i}$ of the $i$ th membrane node is solved by discretizing equation (11) via a second order finite difference method.

The fluid-structure coupling. The fluid and structure are coupled via two coupling conditions at the fluid-structure interface: (1) continuity of velocity, i.e., the no-slip condition, (Kinematic Coupling 
Condition), and (2) balance of forces (Dynamic Coupling Condition). Namely, the balance of forces imposed via the second Newton's law of motion says that the elastodynamics of the fluid-structure interface is driven by the jump in the normal fluid stress across the interface and the external forces acting on the interface.

More precisely, if $\gamma$ denotes the swimmer membrane, and $\overline{\mathbf{F}}$ denotes the contact force that the structure feels exerted by the fluid onto the structure (in Lagrangian coordinates), see equation (11), and $\mathbf{F}^{e}$ denotes the external force on the structure that produces peristaltic wave motion, then the two coupling conditions read:

- No-slip: $d \mathbf{r} / d t=\left.\mathbf{u}\right|_{\gamma}$,

- Balance of forces: $\left.(\mathbf{f} J)\right|_{\gamma}=-\overline{\mathbf{F}}=-m \ddot{\mathbf{r}}+\mathbf{F}(\mathbf{r})+\mathbf{F}^{e}$, where $\mathbf{f}$ is the jump in the normal component of the fluid stress (given in Eulerian coordinates), and $J$ is the Jacobian of the transformation from Eulerian to Lagrangian coordinates.

We do not need to specify (f $J)\left.\right|_{\gamma}$ explicitly here because, in the Immersed Boundary Formulation, the conversion from Eulerian to Lagrangian coordinates is done via integral formulations involving the Dirac delta function, as we present below.

The Immersed Boundary Method (IBM) formulation of the coupled FSI problem. The immersed boundary method, developed by Peskin, see e.g., [21, 22, 23], is employed in our study because of its distinguishing features in dealing with the problem of fluid flow interacting with a flexible fluidstructure interface immersed in the fluid. To write the IBM formulation of the problem we denote by $(q, r, s)$ the (Lagrangian) curvilinear coordinates associated with the structure, and introduce $\mathbf{X}(q ; r ; s ; t)$ to be the Cartesian coordinates at time $t$ of the material point whose label is $(q ; r ; s)$. Furthermore, let $m(q, r, s)$ denote the mass density of the material in the sense that $\int_{\gamma} m(q, r, s) d q d r d s$ is the mass of the part of the material defined by $(q, r, s) \in \gamma$. Introduce $\delta(\mathbf{x})$ to denote the three-dimensional Delta function $\delta\left(x_{1}\right) \delta\left(x_{2}\right) \delta\left(x_{3}\right)$, where $x_{1}, x_{2}, x_{3}$ are the Cartesian components of $\mathbf{x}$. Then the IBM formulation of our FSI problem reads:

$$
\begin{gathered}
\rho\left(\frac{\partial \mathbf{u}}{\partial t}+\mathbf{u} \cdot \nabla \mathbf{u}\right)=-\nabla p+\mu \Delta \mathbf{u}+\mathbf{f}, \\
\nabla \cdot \mathbf{u}=0, \\
\mathbf{f}(\mathbf{x}, t)=\int \overline{\mathbf{F}}(q, r, s, t) \delta(\mathbf{x}-\mathbf{X}(q, r, s, t)) d q d r d s, \\
\frac{d \mathbf{X}}{d t}=\mathbf{U}(\mathbf{X}(q, r, s, t), t)=\int \mathbf{u}(\mathbf{x}, t) \delta(\mathbf{x}-\mathbf{X}(q, r, s, t)) d \mathbf{x},
\end{gathered}
$$

where

$$
-\overline{\mathbf{F}}=-m \ddot{\mathbf{X}}+\mathbf{F}(\mathbf{X})+\mathbf{F}^{e} .
$$

Notice that in this formulation the conversion from Eulerian to the Lagrangian coordinates is done via the integral formulations (14) and (15) and so no explicit calculation of the Jacobian of the transformation $J$ is necessary. The fluid equations (12) and (13) are written entirely in Eulerian form, while the structure equation (16) is written entirely in Lagrangian formulation. The influence of the structure onto the fluid is included as an external force in the fluid equation (12), while the influence of the fluid onto the structure is felt via the no-slip condition (15).

\subsection{Numerical Solver}

We implemented the Immersed Boundary Method to resolve the fluid-structure interaction, and the Fictitious Domain Method to solve the fluid equations. Lagrange multipliers were employed to enforce 
the incompressibility condition and the no-slip condition at the outer, rigid cylinder wall. This approach was recently developed in [25] for the simulation of red blood cells interacting with an incompressible, viscous, Newtonian fluid in a straight cylindrical tube with rigid walls.

The problem is numerically semi-discretized in time using the time discretization via Lie operator splitting. This approach separates the entire FSI problem into "modules", which can easily be modified or dropped if necessary. For example, the advection module is simply ignored (dropped) in the case of Stokes flow without any additional modifications in the fluid solver. The time-discretization via Lie splitting for our Navier-Stokes FSI problem has 4 modules. The scheme starts by considering a certain given velocity and structure position (from the previous time step). In the first module this given fluid flow from the previous time step is modified to enforce the incompressibility condition by solving a degenerate quasi-Stokes problem, see (26). In the second step, this new fluid velocity is used to calculate the new location of the structure by enforcing the no-slip condition (the kinematic coupling condition (15)), i.e., by updating the velocity of the swimmer to be equal to the velocity of the fluid from where the new location of the swimmer is calculated. In this second step, the force exerted by the swimmer onto the fluid (16) is also calculated and used in the next step to solve the momentum equation (12) for the fluid problem, which includes the dynamic coupling condition (14) via force $\mathbf{f}$ that the fluid feels from the structure. In the last step, the momentum equation (12) for the fluid flow is solved. To separate between the advection and diffusion effects in the fluid problem, this last step is split into two modules: the advection module, see equation (28), and the diffusion module, see (29). The advection module can easily be dropped when the Stokes, instead of the Navier-Stokes problem is solved, which is the case with our low-Reynolds number swimmer. The result of these 4 modules is the updated fluid velocity, fluid pressure, and the position of the immersed swimmer during one time step.

The spatial discretization associated with the Immersed Boundary Method formulation (12)-(16) usually incorporates two spatial grids, one for the Eulerian (fluid) variables, and one for the Lagrangian (structure) variables. The Eulerian grid will be based on the finite element method discretization of the fluid domain, which we discuss in more detail below. At this point we specify that we will be using a uniform (Eulerian) finite element mesh, with the mesh size $h$. Based on this grid size $h$, we denote by $g_{h}$ the corresponding 3D Eulerian grid associated with the fluid flow approximation. The corresponding 2D Lagrangian (structure) grid will be denoted by $G_{h}$. Its dependence on $h$ is specified below.

\subsubsection{The Lagrangian Grid for the Discretized Immersed Boundary Method}

The spatial (Lagrangian) discretization of the deformable structure will be performed by discretizing the deformable structure (i.e., the immersed swimmer) via a set of boundary nodes $\mathbf{X}=\left(X_{1}, X_{2}, X_{3}\right)$. These are identified with the nodes $\mathbf{r}_{i}$ in the definition of the swimmer model, described in Section 2.2, where the nodes correspond to the ends of the (nonlinearly) elastic springs. To avoid leaks, we impose that

$$
|\mathbf{X}(q+\Delta q, r, s, t)-\mathbf{X}(q, r, s, t)|<\frac{h}{2} .
$$

The same must hold of $r$ and $s$.

The dynamic and kinematic coupling conditions (14) and (15) are discretized as follows. First, a three-dimensional discrete $\delta$ function $D_{h}(\mathbf{X}-\mathbf{x})$ is introduced:

$$
D_{h}(\mathbf{X}-\mathbf{x})=\delta_{h}\left(X_{1}-x_{1}\right) \delta_{h}\left(X_{2}-x_{2}\right) \delta_{h}\left(X_{3}-x_{3}\right),
$$

where $\delta_{h}$ denotes the one-dimensional discrete $\delta$ function

$$
\delta_{h}(\mathbf{z})= \begin{cases}\frac{1}{8 h}\left[3-2|\mathbf{z}| / h+\sqrt{1+4|\mathbf{z}| / h-4(|\mathbf{z}| / h)^{2}}\right], & |\mathbf{z}| \leq h, \\ \frac{1}{8 h}\left[5-2|\mathbf{z}| / h-\sqrt{-7+12|\mathbf{z}| / h-4(|\mathbf{z}| / h)^{2}}\right], & h \leq|\mathbf{z}| \leq 2 h . \\ 0, & \text { otherwise. }\end{cases}
$$


The dynamic coupling condition (14) is discretized as

$$
\mathbf{f}(\mathbf{x}, t)=\sum_{(q, r, s) \in G_{h}} \overline{\mathbf{F}}(q, r, s, t) D_{h}(\mathbf{X}(q, r, s, t)-\mathbf{x}) \Delta q \Delta r \Delta s \quad \text { for }|\mathbf{X}-\mathbf{x}| \leq 2 h,
$$

where the sum is taken over the Lagrangian grid $G_{h}$, and $-\overline{\mathbf{F}}=\mathbf{F}-m \frac{\partial^{2} \mathbf{X}}{\partial t^{2}}+\mathbf{F}^{\mathrm{e}}$.

The calculation of the structure velocity $\mathbf{U}(\mathbf{X})$ via the kinematic coupling condition (15) is discretized as follows:

$$
\mathbf{U}(\mathbf{X}(q, r, s, t))=\sum_{\mathbf{x} \in g_{h}} h^{3} \mathbf{u}(\mathbf{x}) D_{h}(\mathbf{X}(q, r, s, t)-\mathbf{x}) \quad \text { for }|\mathbf{X}-\mathbf{x}| \leq 2 h,
$$

where the sum is taken over the Eulerian (fluid) grid $g_{h}$.

When the time discretization is introduced later, we will use this equation to update the position of the immersed boundary by calculating

$$
\mathbf{X}_{t+\Delta t}=\mathbf{X}_{t}+\Delta t \mathbf{U}\left(\mathbf{X}_{t}\right)
$$

\subsubsection{Numerical Approximation of the Fluid Sub-problem: Fictitious Domain with Lagrange Multipliers}

To define the fictitious domain formulation for the fluid problem (1)-(4), or problem (12), (13) in the cylindrical tube $\omega$, we introduce a larger, "simpler", "fictitious" rectangular fluid domain $\Omega \subset R^{3}$, see Figure 2, where $\Omega$ is a rectangular parallelepiped whose width is equal to the diameter of the fluid domain $\omega$, and whose length is equal to $L$. We will solve the fluid equations everywhere in $\Omega$, with a constraint that the fluid velocity outside of $\omega$, namely in the "complement" $\omega^{c}:=\Omega \backslash \bar{\omega}$, remains zero at all times. This will enforce, among other things, the no-slip condition $\mathbf{u}=\mathbf{0}$ at the fixed cylinder boundary $\partial \omega$.

To enforce the zero fluid velocity in $\omega^{c}$, and to enforce the incompressibility condition, we use the approach based on Lagrange multipliers. The fictitious domain formulation with Lagrange multipliers (DLM/FD formulation) for the fluid problem (1)-(4) in a cylindrical tube $\omega$ reads as follows:

For a.e. $t>0$, find $\mathbf{u}(t) \in W_{0, P}, p(t) \in L_{0}^{2}, \boldsymbol{\mu}_{\omega^{c}} \in \boldsymbol{\Lambda}_{\omega^{c}}$ such that

$$
\begin{aligned}
& \left\{\begin{array}{l}
\rho \int_{\Omega}\left[\frac{\partial \mathbf{u}}{\partial t}+(\mathbf{u} \cdot \nabla) \mathbf{u}\right] \cdot \mathbf{v} \mathrm{d} \mathbf{x}+\mu \int_{\Omega} \nabla \mathbf{u}: \nabla \mathbf{v} \mathrm{d} \mathbf{x}-\int_{\Omega} p \nabla \cdot \mathbf{v} \mathrm{d} \mathbf{x} \\
=\int_{\Omega} \mathbf{f} \cdot \mathbf{v} \mathrm{d} \mathbf{x}+<\boldsymbol{\lambda}_{\omega^{c}}, \mathbf{v}>_{\omega^{c}}, \quad \forall \mathbf{v} \in W_{0, P},
\end{array}\right. \\
& \int_{\Omega} q \nabla \cdot \mathbf{u}(t) \mathrm{d} \mathbf{x}=0, \quad \forall q \in L^{2}(\Omega), \\
& <\boldsymbol{\mu}_{\omega^{c}}, \mathbf{u}(t)>_{\omega^{c}}=0, \quad \forall \boldsymbol{\mu}_{\omega^{c}} \in \boldsymbol{\Lambda}_{\omega^{c}}, \\
& \mathbf{u}(\mathbf{x}, 0)=\mathbf{u}_{0}(\mathbf{x}),
\end{aligned}
$$

where

$W_{0, P}=\left\{\mathbf{v} \mid \mathbf{v} \in\left(H^{1}(\Omega)\right)^{3}, \mathbf{v}=\mathbf{0}\right.$ on four "lateral" sides of $\Omega, \mathbf{v}$ is L-periodic in the $x$ direction $\}$, $L_{0}^{2}=\left\{q \mid q \in L^{2}(\Omega), \int_{\Omega} q \mathrm{~d} \mathbf{x}=0\right\}$,

$\boldsymbol{\Lambda}_{\omega^{c}}=\left\{\boldsymbol{\mu} \mid \boldsymbol{\mu} \in\left(H^{1}\left(\omega^{c}\right)\right)^{3}, \boldsymbol{\mu}\right.$ is periodic in the $x$ direction $\}$.

Here $\boldsymbol{\lambda}_{\omega^{c}}$ is a Lagrange multiplier associated with relation (24), constraining the fluid velocity on $\omega^{c}$ to be equal to zero, and $\langle\cdot, \cdot\rangle_{\omega^{c}}$ is an inner product on $\boldsymbol{\Lambda}_{\omega^{c}}$ (see [20] for more information). Similarly, $p$ is the Lagrange multiplier associated with the divergence free condition (23).

The space discretization. The fictitious fluid domain $\bar{\Omega} \subset R^{3}$ is approximated by tetrahedra of "size" (diameter) $h$, and we denote this Eulerian (fluid) grid with $g_{h}$. Let $P_{i}$ denote the space of polynomials in three variables of degree less than or equal to $i$. To approximate the velocity and pressure $\{\mathbf{u}, p\}$ we use the $P_{1}-i s o-P_{2}$ and $P_{1}$ finite elements, respectively, (see, e.g., [12] (Chapter 5)), for which 
$\mathbf{T}_{h}$ denotes a finite element tetrahedralization of $\bar{\Omega}$ for velocity, and $\mathbf{T}_{2 h}$ denotes the twice coarser tetrahedralization used for the pressure. Our mesh $g_{h}$ is identified with the tetrahedralization $\mathbf{T}_{h}$. The following finite-dimensional spaces are introduced to approximate the spaces in problem (22)-(25):

$W_{h}=\left\{\mathbf{v}_{h}\left|\mathbf{v}_{h} \in C^{0}(\bar{\Omega})^{3}, \mathbf{v}_{h}\right|_{T} \in\left(P_{1}\right)^{3}, \forall T \in \mathbf{T}_{h}, \mathbf{v}_{h}\right.$ is periodic in the $x$ direction with period $\left.L\right\}$,

$W_{0, h}=\left\{\mathbf{v}_{h} \mid \mathbf{v}_{h} \in W_{h}, \mathbf{v}_{h}=\mathbf{0}\right.$ on four "lateral" sides of $\left.\Omega\right\}$,

$L_{h}^{2}=\left\{q_{h}\left|q_{h} \in C^{0}(\bar{\Omega}), q_{h}\right|_{T} \in P_{1}, \forall T \in \mathbf{T}_{2 h}, q_{h}\right.$ is periodic in the $x$ direction with period $\left.L\right\}$,

$L_{0, h}^{2}=\left\{q_{h} \mid q_{h} \in L_{h}^{2}, \int_{\Omega} q_{h} d x=0\right\}$.

A finite dimensional space approximation of $\boldsymbol{\Lambda}_{\omega^{c}}$, denoted by $\boldsymbol{\Lambda}_{\omega^{c}{ }_{h}}$, is defined as follows: let $\left\{\mathbf{x}_{i}\right\}_{i=1}^{M}$ be a set of points from $\overline{\omega^{c}}$ which cover $\overline{\omega^{c}}$ (uniformly, for example). Then

$$
\boldsymbol{\Lambda}_{\omega^{c} h}=\left\{\boldsymbol{\mu}_{h} \mid \boldsymbol{\mu}_{h}=\sum_{i=1}^{M} \boldsymbol{\mu}_{i} \delta\left(\mathbf{x}-\mathbf{x}_{i}\right), \boldsymbol{\mu}_{i} \in \mathbf{R}^{3}, \forall i=1, \cdots, N\right\}
$$

where $\delta(\cdot)$ is the Dirac measure at $\mathbf{x}=\mathbf{0}$. Finally, we use $\langle\cdot, \cdot\rangle_{\omega_{h}(t)}$ to denote

$$
<\boldsymbol{\mu}_{\omega^{c} h}, \mathbf{v}_{h}>_{\omega^{c} h}=\sum_{i=1}^{M} \boldsymbol{\mu}_{i} \cdot \mathbf{v}_{h}\left(\mathbf{x}_{i}\right), \forall \boldsymbol{\mu}_{\omega^{c}{ }_{h}} \in \boldsymbol{\Lambda}_{\omega^{c}{ }^{c}}, \mathbf{v}_{h} \in W_{0, h} .
$$

Remark 1. A typical choice of the points for $\boldsymbol{\Lambda}_{\omega^{c} h}$ is to take the grid points of the velocity mesh internal to the region $\overline{\omega^{c}}$ and whose distance to the surface of the cylinder $\omega$ is greater than, e.g. $h$, and to complete selected points from the surface of the cylinder $\omega$. In practice, we have chosen $\Omega$ with its square cross section slightly larger than the cross section of the cylinder $\omega$ in order to have collocation points between the surface of the cylinder $\omega$ and $\Gamma$ so that the enforcement of the constraint over $\overline{\omega^{c}}$ can be done much more easily.

\subsubsection{The time discretization via Lie splitting for the coupled FSI problem}

As mentioned earlier, the full FSI problem, given by (12)-(16), is discretized in time by using the Lie's Scheme. See [12] for the definition and details about the Lie scheme. To solve problem (12)-(16) on the time interval $(0, T)$, the time-discretization step $\Delta t$ is introduced, and the interval is split into $N$ sub-intervals $\left(\left(0, t_{1}\right), \cdots,\left(t_{n}, t_{n+1}\right), \cdots,\left(t_{N-1}, t_{N}\right)\right)$. On each sub-interval $\left(t_{n}, t_{n+1}\right)$ a sequence of four sub-problems is solved, where each sub-problem uses for the initial data at $t=t_{n}$ the solution of the just calculated sub-problem, evaluated at time $t=t_{n+1}$. More precisely, our Lie splitting scheme is defined as follows.

Let $\mathbf{u}^{0}=\mathbf{u}_{0}$ be given (initial data for velocity). For $n \geq 0$, let $\mathbf{u}^{n}, \mathbf{X}^{n}$ be known, compute $\mathbf{u}^{n+1}, \mathbf{X}^{n+1}, p^{n+1}$ via the following four fractional steps:

(1) Enforce the Incompressibility Condition, i.e., Conservation of Mass. Find the fluid velocity and pressure by solving on $\left(t^{n}, t^{n+1}\right)$ :

$$
\left\{\begin{array}{l}
\rho \int_{\Omega} \frac{\mathbf{u}^{n+1 / 4}-\mathbf{u}^{n}}{\triangle t} \cdot \mathbf{v} d \mathbf{x}-\int_{\Omega} p^{n+1 / 4}(\nabla \cdot \mathbf{v}) d \mathbf{x}=0 \forall \mathbf{v} \in W_{0, h}, \\
\int_{\Omega} q \nabla \cdot \mathbf{u}^{n+1 / 4} d \mathbf{x}=0 \forall q \in L_{h}^{2} \\
\mathbf{u}^{n+1 / 4} \in W_{0, h}^{n+1}, p^{n+1 / 4} \in L_{0, h}^{2}
\end{array}\right.
$$

Since the fluid pressure is not going to change anymore during this time step, set $p^{n+1}=p^{n+1 / 4}$.

(2) Update the position of the immersed structure and the force it exerts on the fluid. Update the position of the immersed structure based on $\mathbf{u}^{n+1 / 4}$ by solving on $\left(t^{n}, t^{n+1}\right)$

$$
\mathbf{X}^{n+1 / 4}=\mathbf{X}^{n}+\Delta t \mathbf{U}^{n+1 / 4}
$$

where $\mathbf{U}^{n+1 / 4}$ is obtained using (20) with the just calculated fluid velocity $\mathbf{u}^{n+1 / 4}$. Since $\mathbf{X}$ will not change in this time-step anymore, we set $\mathbf{X}^{n+1}:=\mathbf{X}^{n+1 / 4}$. Then compute the force $\mathbf{f}^{n+1}$ by using (9) and (19). 
(3) Account for fluid advection. Update the fluid velocity by solving on $\left(t^{n}, t^{n+1}\right)$ :

$$
\left\{\begin{array}{l}
\int_{\Omega} \frac{\partial \mathbf{u}(\mathbf{t})}{\partial t} \cdot \mathbf{v} d \mathbf{x}+\int_{\Omega}\left(\mathbf{u}^{n+1 / 4} \cdot \nabla\right) \mathbf{u}(\mathbf{t}) \cdot \mathbf{v} d \mathbf{x}=0 \text { on }\left(t^{n}, t^{n+1}\right) \forall \mathbf{v} \in W_{0, h}, \\
\mathbf{u}\left(t^{n}\right)=\mathbf{u}^{n+1 / 4} \\
\mathbf{u}(t) \in W_{0, h} \text { on }\left(t^{n}, t^{n+1}\right),
\end{array}\right.
$$

and set $\mathbf{u}^{n+3 / 4}=\mathbf{u}\left(t^{n+1}\right)$.

(4) Account for fluid dissipation including the force exerted by the structure onto the fluid. Update the fluid velocity by solving on $\left(t^{n}, t^{n+1}\right)$ :

$$
\left\{\begin{array}{l}
\rho \int_{\Omega} \frac{\mathbf{u}^{n+1}-\mathbf{u}^{n+3 / 4}}{\triangle t} \cdot \mathbf{v} d \mathbf{x}+\mu \int_{\Omega} \nabla \mathbf{u}^{n+1}: \nabla \mathbf{v} d \mathbf{x}=\int_{\Omega} \mathbf{f}^{n+1} \cdot \mathbf{v} d \mathbf{x}+<\boldsymbol{\lambda}_{\omega^{c}{ }_{h}}, \mathbf{v}>_{\omega^{c} h} \quad \forall \mathbf{v} \in W_{0, h}, \\
<\boldsymbol{\mu}_{\omega^{c} h}, \mathbf{u}^{n+1}>_{\omega^{c} h}=0 \forall \boldsymbol{\mu}_{\omega^{c} h} \in \boldsymbol{\Lambda}_{\omega^{c} h}, \\
\mathbf{u}^{n+1} \in W_{0, h}, \boldsymbol{\lambda}_{\omega^{c}{ }_{h}} \in \boldsymbol{\Lambda}_{\omega^{c}{ }_{h}}
\end{array}\right.
$$

These four sub-steps define the new values for the fluid velocity, pressure, and structure position: $\left(\mathbf{u}^{n+1}, p^{n+1}, \mathbf{X}^{n+1}\right)$. Change $n=n+1$ and return to step (1).

The degenerate quasi-Stokes problem (26) is solved by a preconditioned conjugate gradient method (e.g., see [12]), in which discrete elliptic problems from the preconditioning are solved by a matrix-free fast solver from FISHPAK by Adams et al. in [1]. The advection problem (28) for the velocity field is solved by a wave-like equation method as in [7] and [8]. The time-dependent Stokes problem (29) with a source term can be solved by a conjugate gradient method (see, e.g., [20]).

This method is first-order accurate in time [12]. A symmetrization of the algorithm increases the accuracy to second-order, giving rise to the Strang splitting scheme [8].

We conclude this section by noting that to simulate the low Reynolds number (Stokes) flow, we simply skip step (3) in this scheme. We compared the results obtained with the Navier-Stokes and the Stokes solver and did not observe any difference worth reporting, as expected.

\section{Simulation results and discussion}

We considered the influence of the following non-dimensional parameters on the propulsion of the swimmer caused by a peristaltic motion of the swimmer membrane immersed in a fluid at low Reynolds numbers:

- The ratio $\epsilon$ between the swimmer reference radius $R_{0}$ and reference length $L$;

- The ratio $\lambda$ between the swimmer (final) deformed radius $R_{f}$ and its reference radius $R_{0}$, i.e., the amplitude of the peristaltic wave.

We first focus on one cycle of force application (one peristaltic wave contraction), and then consider multiple peristaltic contractions of the swimmer during a fixed time interval, which we present in Section 4.5.

Basic parameter values. The parameter values used in all the simulations are as follows: The bending constant is $k_{b}=1.0 \times 10^{-15} \mathrm{~N}$ and the spring constant is $k_{s}=5.5 \times 10^{-8} \mathrm{~N} / \mathrm{m}$. The swimmer is suspended in an incompressible, viscous Newtonian fluid with density $\rho=1.00 \mathrm{~g} / \mathrm{cm}^{3}$ and dynamic viscosity $\mu=0.012 \mathrm{~g} /(\mathrm{cm} \mathrm{s})$. The computational domain is a horizontal tube with $2 \mu \mathrm{m}$ in length and $0.25 \mu \mathrm{m}$ in radius. Periodic boundary conditions are imposed in the horizontal $x$ direction. The velocity of the fluid flow is zero everywhere initially. The time step $\Delta t=0.01 \mathrm{~ms}$ over the maximum time interval of $300 \mu s$.

Numerical convergence results were performed both in space and in time, and we report below the investigations performed with the mesh size and time step that correspond to the converged results. 
Application of force. Each swimmer surface is divided into $N$ segments. The length of the first segment on the right, i.e., the segment $\left(l_{N-1}, l_{N}\right)$, is the shortest in order to minimize the initial backward motion of the swimmer, which takes place after the application of force to the right-most segment $\left(l_{N-1}, l_{N}\right)$. In the simulations presented below, the length of the first segment is the same for all swimmers, and it is equal to the equilibrium length of the horizontal springs connecting different nodes in the swimmer. The remaining $N-1$ segments are all bigger and of equal length, which is 3 or 4 times the length of the first segment on the right $\left(l_{N-1}, l_{N}\right)$, depending on the length of the swimmer.

To produce a peristaltic wave, shown in Figure 4, traveling from right to left, with an amplitude $R_{0}-R_{f}$ determined by the "squeezed" radius $R_{f}$, the following strategy in applying the force to the swimmer surface is applied:

1. The force $\mathbf{F}_{\text {ext }} / N_{n}$, where $N_{n}$ is the total number of nodes in the swimmer, and $\mathbf{F}_{\text {ext }}=0.1 p N$ is applied to each node of the first segment on the right $\left(l_{N-1}, l_{N}\right)$, for the time duration of $t_{1}$ seconds. The number $t_{1}$ depends on the targeted final radius of the swimmer.

2. For each $i=2, \cdots, N-1$ the force $\mathbf{F}_{\text {ext }} / N_{n}$ is applied to each node of the segment $\left(l_{N-i-1}, l_{N-i}\right)$ for $t_{i}$ seconds. At the same time the radius of the previous segment is checked, and the following force is applied to the previous segment(s):

(a) $\mathbf{F}_{\text {ext }} / N$, if the deformed radius of the swimmer is still bigger than the desired "final" radius $R_{f}$ plus some tolerance $\delta$;

(b) $-\mathbf{F}_{\text {ext }} / N$, if the deformed radius is below the desired radius $R_{f}$ minus some tolerance $\delta$;

(c) 0 , if the deformed radius is within $\delta$ tolerance of the desired radius $R_{f}$.

Once the wave reaches the left end, we could release all the force and wait for the swimmer to re-assume the original equilibrium position. This, however, may take too long, and we opt for a strategy that will speed up the recovery of the original equilibrium position of the swimmer by applying interior force to the swimmer surface in the negative radial direction. This allows rapid recovery and enables the next contraction cycle to be applied sooner.

To recover the original, equilibrium position of the swimmer the following strategy is applied:

1. Apply the force $-\mathbf{F}_{\text {ext }} / N_{n}$ to the node if the radius of the swimmer at that node is smaller than $0.5 R_{0}$;

2. Apply the force $-0.5 \mathbf{F}_{\text {ext }} / N_{n}$ to the node if the radius of the swimmer at that node is between $0.5 R_{0}$ and $0.8 R_{0}$;

3. Apply the force $-0.1 \mathbf{F}_{\text {ext }} / N_{n}$ to the node if the radius of the swimmer at that node is between $0.8 R_{0}$ and $R_{0}-\delta$;

4. Apply the force $0.1 \mathbf{F}_{\text {ext }} / N_{n}$ to the node if the radius of the swimmer at that node is bigger than $R_{0}+\delta$

5. Apply zero force to the node if the radius of the swimmer at that node is within $\delta$ tolerance of $R_{0}$.

We tested this strategy on a "dry swimmer", i.e., a swimmer not immersed in the fluid, modeled by the following viscoelastic model:

$$
m \ddot{\mathbf{r}}+\nu \dot{\mathbf{r}}-\mathbf{F}(\mathbf{r})=\mathbf{F}^{e x t},
$$

in place of equation (5), where $\nu$ is some viscoelasticity constant simulating the damping effects by a viscous fluid. Figure 4 shows an example of the generated peristaltic wave in a dry swimmer of reference length $L=0.125 \mu \mathrm{m}$ and reference radius $R_{0}=0.4 \mu \mathrm{m}$.

\subsection{The influence of the ratio $\epsilon$ between the swimmer reference radius $R_{0}$ and reference length $L$}

To study the influence of the parameter $\epsilon=R_{0} / L$ on the propulsion of the swimmer immersed in fluid, we fixed the reference radius $R_{0}=0.125 \mu \mathrm{m}$ and changed the length $L$ of the swimmer to the following three values: 

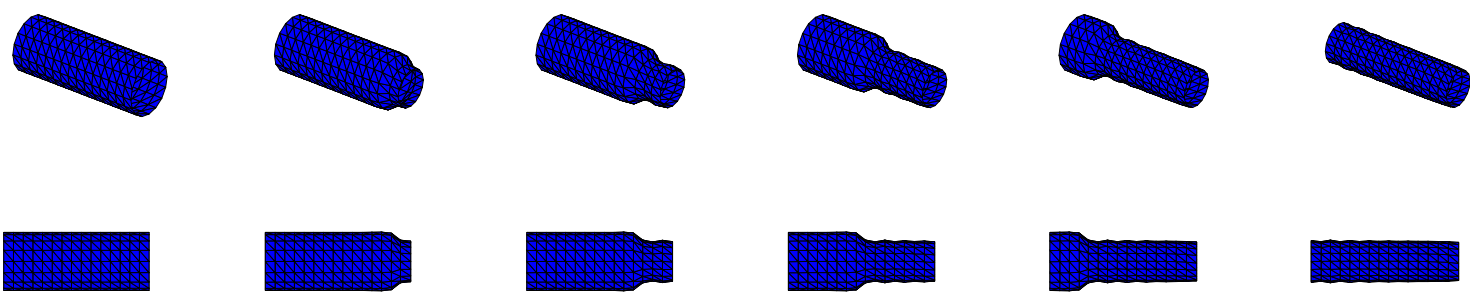

Figure 4: Snapshots of the deformation of the swimmer (top) and associated side view (bottom).

- $L^{1}=0.2 \mu m, \epsilon_{1}=0.625$,

- $L^{2}=0.4 \mu m, \epsilon_{2}=0.3125$,

- $L^{3}=0.8 \mu m, \epsilon_{3}=0.1563$.

In each of the three cases listed above we deformed the swimmer to the same "final" radius $R_{f}=0.55 R_{0}$ producing a peristaltic wave in the swimmer wall traveling at roughly the same speeds for all three swimmers. See Figure 5.
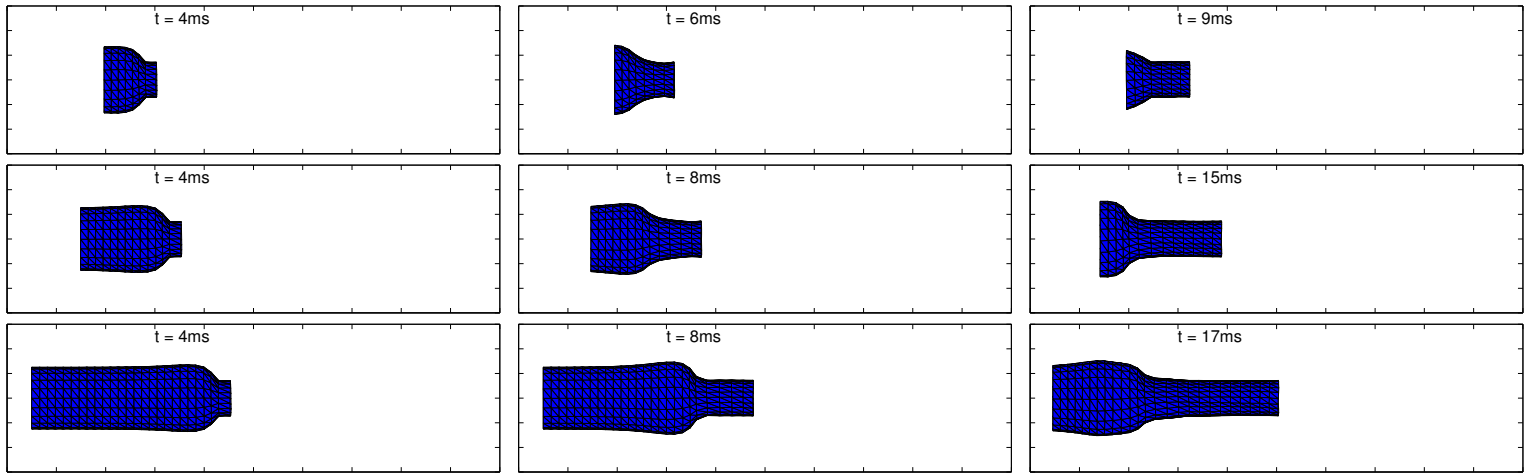

Figure 5: Side view of the configuration of 3 swimmers with the same reference radius $R_{0}=0.125 \mu m$, and different reference lengths: $L^{1}=0.2 \mu m$ (top), $L^{2}=0.4 \mu m$ (middle), and $L^{3}=0.8 \mu m$ (bottom). The snap shots at three different (representative) times are taken for each swimmer showing propagation of the propulsion wave. As the wave reaches the end of the swimmer, the final configuration (not shown here) becomes a straight cylinder with deformed radius $R_{f}=0.55 R_{0}$.

One can notice in Figure 5 that the longer the swimmer, the longer it takes the peristaltic wave to reach the end of the swimmer. We tracked the history of each swimmer's middle point, i.e., the center of mass of the reference swimmer configuration. The distance traversed by each swimmer's center of mass is shown in Figure 6 (left). The red curve in Figure 6 (left) shows that maximum distance traveled by the swimmer with length $L^{3}=0.8 \mu \mathrm{m}$ is $0.08 \mu \mathrm{m}$, the black curve shows that that maximum distance traveled by the swimmer with length $L^{2}=0.4 \mu \mathrm{m}$ is $0.05 \mu \mathrm{m}$, and the blue curve shows that that maximum distance traveled by the swimmer with length $L^{1}=0.2 \mu m$ is $0.034 \mu m$. The history of the total energy of each swimmer is shown in Figure 6 (right). The results shown in Figure 6 are used to draw the following conclusions about the influence of $\epsilon=R_{0} / L$ on the distance traveled by the swimmer.

Conclusions. We observed, as expected, that at the end of one cycle of the traveling wave sweeping the swimmer from right to left, the swimmer that moves the farthest, shown in Figure 6 , was the swimmer with the largest length, displacing the largest amount of fluid in one cycle. The "tails" in this 

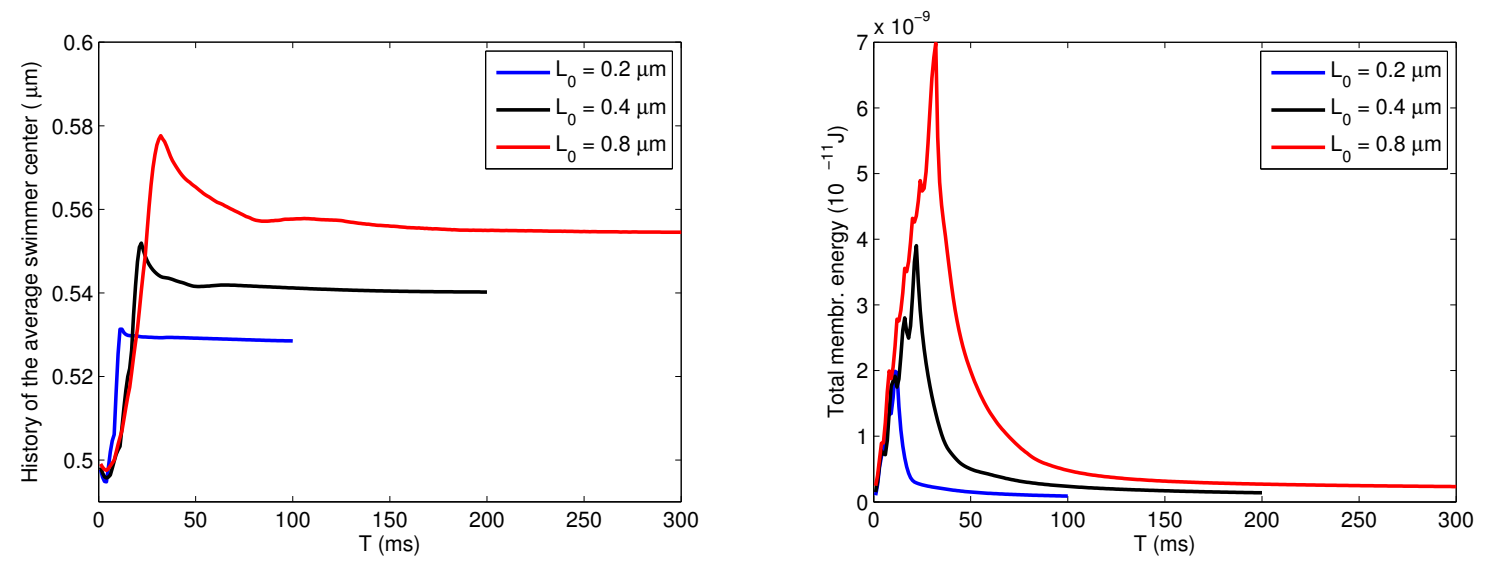

Figure 6: History of motion of swimmer center (left) and of total energy (right) for the three swimmers shown in Figure 5.

figure correspond to the location of the center of the swimmer during recovery to the equilibrium position when the total energy of the swimmer becomes close to zero within some $\delta$ tolerance. The same tolerance measuring the distance to zero energy was used for all swimmers. From Figure 6 (right), which shows the history of the total energy for each swimmer, one can see that the longer the swimmer, the longer it takes the swimmer to reach its equilibrium position. This will be important when multiple cycles of force application are considered, discussed in Section 4.5 below.

\subsection{The influence of the ratio $\lambda$ between the deformed radius $R_{f}$ and the reference radius $R_{0}$}

We consider a swimmer with the reference radius $R_{0}=0.125 \mu \mathrm{m}$ and reference length $L=0.4 \mu \mathrm{m}$. A force is applied to the surface of the swimmer in such a way to produce a peristaltic wave, with three different amplitudes by deforming the swimmer to three different "final" radii, see Figure 7:

$$
R_{f}^{1}=0.35 R_{0}, \quad R_{f}^{2}=0.55 R_{0}, \quad R_{f}^{3}=0.82 R_{0} .
$$
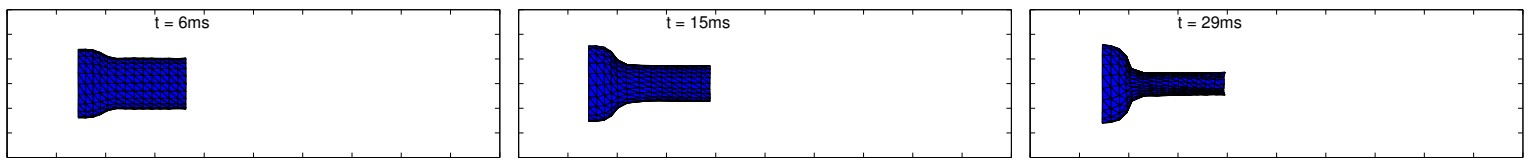

Figure 7: Side view of the configuration of 3 swimmers with the same reference radius $R_{0}=0.125 \mu m$, and same reference length $L=0.4 \mu \mathrm{m}$, deformed to three different "final" radii: $R_{f}^{1}=0.35 R_{0}$ (left), $R_{f}^{2}=0.55 R_{0}$ (middle), and $R_{f}^{3}=0.82 R_{0}$ (right). The three snap shots were taken just before the peristaltic wave reaches the end of the cylinder for each swimmer.

Figure 8 (left) shows the location of the swimmer center versus time for the three swimmers, while Figure 8 (right) show the total energy versus time for the three swimmers. As before, the tails in both figures correspond to the recovery to the equilibrium position when the total energy of the swimmer becomes close to zero within some $\delta$ tolerance.

Conclusions. We observe from Figure 8 that the swimmer that deforms to the smallest radius during the peristaltic wave motion, i.e., for which the amplitude of the peristaltic wave is largest, moves farthest during one cycle of the application of force producing the peristaltic traveling wave, as expected. However, Figure 8 also shows that to produce a peristaltic wave with large amplitude, it takes longer to deform the swimmer than the one with smaller amplitude. Similarly, the swimmers experiencing large 

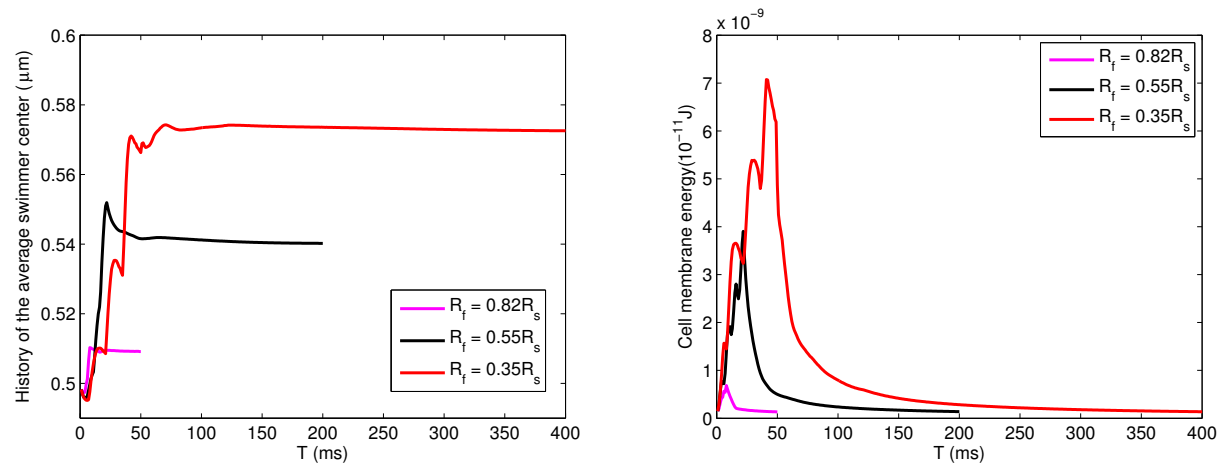

Figure 8: History of the swimmer center as a function of time (left) and the associated total energy of the swimmer as a function of time (right).

deformations take longer to get back to their original equilibrium state. This is because of the viscous effects of the surrounding fluid, and due to the "added mass effect" exerted onto the deforming swimmer by the surrounding fluid. This will be discussed in more detail in Section 4.5, where multiple swimmer contractions are studied.

To get a deeper insight into how different parameters in the problem influence the motion of peristaltic swimmers, we derive a reduced, 1D model approximating fluid-structure interaction between a slender elastic swimmer and the surrounding low Reynolds number fluid. We will use the 1D model to see if we can predict distances traveled by the 3D swimmers studied in Sections 3.1 and 3.2, and to better understand the mechanisms and combinations of parameters that influences motion of elastic swimmers. The derivation of the $1 \mathrm{D}$ reduced model is presented next.

\section{A 1D Reduced Model for FSI between cylindrical swimmers and creeping flow}

We derive the so called "lubrication model" for an axially symmetric flow of a "creeping fluid" interacting with a slender elastic hollow cylinder modeling a micro-swimmmer. The resulting model is a low-Reynolds number version of the 1D FSI model studied in detail in [6].

\subsection{The axially symmetric $3 D$ model}

We begin by considering a cylindrical swimmer interacting with an incompressible, viscous, Newtonian fluid, producing an axially symmetric flow, so that the Navier-Stokes equations for an incompressible, viscous Newtonian fluid in cylindrical coordinates can be used to model the fluid flow. We will be using $(r, z, \theta)$ to denote the cylindrical coordinates, where $z$ is aligned with the horizontal axis of the cylinder. It will be assumed that the elastic structure, i.e., the swimmer, behaves as a linearly elastic membrane, i.e., that the displacement and displacement gradients are relatively small. As we shall see below, this will be a good approximation of our nonlinear swimmer problem for relatively small displacements, as expected. In summary, the basic assumptions for the 1D model will be:

- The swimmer is "slender", i.e., $\frac{R_{0}}{L}=\epsilon<<1$;

- The flow is slow, i.e., the Reynolds number is small, i.e., $R e=\mathcal{O}\left(\epsilon^{2}\right)$;

- The flow is axially symmetric so that nothing in the problem depends on the azimuthal variable $\theta$;

- The overall, global elastic behavior of the swimmer can be modeled using the linear membrane equations with only the radial component of displacement different from zero. 
In regard to the last point, we recall that our swimmer was modeled as a network of nonlinearly elastic springs. This elastic network leads to a global, emergent elastic behavior of the entire cylinder. We are assuming that this global elastic behavior of the swimmer can be modeled using the linear membrane equations. In cylindrical coordinates, assuming only radial displacement to be different from zero, the elastic membrane equation reads

$$
m \frac{\partial^{2} R(z, t)}{\partial t^{2}}+\frac{4 E h}{3 R_{0}}\left(\frac{R(z, t)}{R_{0}}-1\right)=f-f_{\text {ref }} .
$$

Here, $m=\rho_{s} h$ where $\rho_{s}$ and $h$ are the structure density and thickness, $R(z, t)$ is the radius of the deformed cylinder (membrane) wall, $R_{0}$ is the reference radius of the cylinder, $E$ is the Youngs modulus, $h$ is the membrane thickness, and $f$ is the force density. At $f=f_{r e f}$ the radius of the cylinder becomes the reference radius $R_{0}$. This defines our structure equation. This equation is related to the swimmer model (5) by assuming zero axial displacement and a linearization of the nonlinear term $\mathbf{F}(\mathbf{r})$.

The fluid flow, as mentioned before, is governed by the Navier-Stokes equations for an incompressible, viscous fluid. Due to the assumption that the flow is axially symmetric, nothing in the problem depends on the azimuthal variable $\theta$, and the fluid velocity only has the radial and axial components, which both depend only on $(t, r, z)$ :

$$
\boldsymbol{u}(t, r, z)=\left(u_{z}(t, r, z), u_{r}(t, r, z)\right) .
$$

The Navier-Stokes equations in cylindrical coordinates assuming axially symmetric flow read:

$$
\begin{aligned}
\frac{\partial u_{z}}{\partial t}+u_{r} \frac{\partial u_{z}}{\partial r}+u_{z} \frac{\partial u_{z}}{\partial z}+\frac{1}{\rho} \frac{\partial p}{\partial z} & =\frac{\mu}{\rho}\left[\frac{\partial^{2} u_{z}}{\partial r^{2}}+\frac{1}{r} \frac{\partial u_{z}}{\partial r}+\frac{\partial^{2} u_{z}}{\partial x^{2}}\right] \\
\frac{\partial u_{r}}{\partial t}+u_{r} \frac{\partial u_{r}}{\partial r}+u_{z} \frac{\partial u_{r}}{\partial z}+\frac{1}{\rho} \frac{\partial p}{\partial r} & =\frac{\mu}{\rho}\left[\frac{\partial^{2} u_{r}}{\partial r^{2}}+\frac{1}{r} \frac{\partial u_{r}}{\partial r}-\frac{u_{r}}{r^{2}}+\frac{\partial^{2} u_{r}}{\partial z^{2}}\right] \\
\frac{\partial u_{z}}{\partial z}+\frac{1}{r} \frac{\partial\left(r u_{r}\right)}{\partial r} & =0
\end{aligned}
$$

The corresponding fluid Cauchy stress tensor is given by the following:

$$
\boldsymbol{\sigma}=\left[\begin{array}{cc}
-p+2 \mu \frac{\partial u_{z}}{\partial z} & \mu\left(\frac{\partial u_{r}}{\partial z}+\frac{\partial u_{z}}{\partial r}\right) \\
\mu\left(\frac{\partial u_{r}}{\partial z}+\frac{\partial u_{z}}{\partial r}\right) & -p+2 \mu \frac{\partial u_{r}}{\partial r}
\end{array}\right]
$$

Fluid-Structure Coupling. We first assume that the cylinder membrane is fixed at the end points and, therefore, cannot move in the horizontal direction. In the local frame of reference of the cylinder, we impose, as usual, the no-slip condition at the cylinder boundary $\gamma$ (continuity of velocities), and the balance of forces acting in the radial direction, i.e., the second Newton's law of motion in the radial direction (recall, we assumed that there is zero longitudinal displacement of the membrane wall relative to the reference configuration of the cylinder):

$$
\begin{aligned}
& (\partial R(z, t) / \partial t, 0)=\left.\left(u_{r}, u_{z}\right)\right|_{\gamma(t)} \\
& \text { (Kinematic condition), } \\
& \left.m \frac{\partial^{2} R(z, t)}{\partial t^{2}}+\frac{4 E h}{3 R_{0}}\left(\frac{R(z, t)}{R_{0}}-1\right)=\left.\left[(\boldsymbol{\sigma} \boldsymbol{n}) \cdot \boldsymbol{e}_{r}\right]\right|_{\gamma(t)} J-\mathbf{F}^{e} \cdot \boldsymbol{e}_{r}, \quad \text { (Dynamic condition }(\mathrm{r})\right) .
\end{aligned}
$$

Here, $\left.\left[(\boldsymbol{\sigma} \boldsymbol{n}) \cdot \boldsymbol{e}_{r}\right]\right|_{\gamma(t)}$ denotes the jump in the radial component of the normal fluid stress exerted on $\gamma(t),-\mathbf{F}^{e} \cdot \boldsymbol{e}_{r}$ is the external force applied to the swimmer in the negative radial direction causing swimmer contraction, and $J$ is the Jacobian of the transformation from Eulerian to Jacobian coordinates: $J=\left(1+\frac{\eta}{R}\right) \sqrt{1+\frac{\partial \eta}{\partial z}}$. The term $\left.\left[(\boldsymbol{\sigma} \boldsymbol{n}) \cdot \boldsymbol{e}_{r}\right]\right|_{\gamma(t)}$ is equal to the radial component of the normal stress external to the cylinder evaluated on $\gamma$, minus the radial component of the normal stress internal to the cylinder evaluated on $\gamma$. As we shall see below, the leading-order approximation of this term will be the 
pressure contribution $-p$, so that this term will become $\left.\left.\left[(\boldsymbol{\sigma} \boldsymbol{n}) \cdot \boldsymbol{e}_{r}\right]\right|_{\gamma(t)} \approx[-p]\right|_{\gamma}=-\left(p_{\text {ext }}-\left.p\right|_{\gamma}\right)$, where $p_{\text {ext }}$ is the pressure external to the cylinder.

Once the cylinder end points are no longer fixed, the cylinder will move in the axial direction because of the effects generated by the displaced mass of the fluid during the peristaltic motion of the cylinder (the rate of change of axial momentum due to the displaced mass) and the viscous drag force exerted by the fluid onto the cylinder wall. Because of the assumption that the membrane does not stretch in the longitudinal direction, all the points on the cylinder will move in the axial direction with the same speed, which we denote by:

$$
W(t) \text { - horizontal velocity of the cylinder. }
$$

We can formulate the dynamic coupling condition in the axial direction similar to the dynamics coupling condition in the radial direction (36) by stating that mass times acceleration in the axial direction of the swimmer is equal to the jump in the axial component of the normal stress across $\gamma(t)$ plus the forces causing propulsion of the swimmer in the axial direction. The force causing the propulsion of the swimmer in the axial direction is equal to the rate of change of mass flux, where the mass flux (or momentum) is equal to

$$
Q=M U \text {. }
$$

Here $M$ is fluid mass and $U$ is the relative fluid velocity with respect to the cylinder, i.e., it is the cross-sectional average of the axial component of velocity of the fluid within the cylinder:

$$
U(z, t)=\frac{2}{R^{2}} \int_{0}^{R} u_{z} r d r .
$$

Since the swimmer velocity is independent of $z$, the forces affecting the motion of the swimmer in the axial direction are averaged with respect to $z$. Thus, the second Newton's law of motion describing the motion of the cylinder in the axial direction is given by:

$$
m \frac{d W(t)}{d t}=\left.\frac{1}{L} \int_{0}^{L}\left[(\boldsymbol{\sigma} \boldsymbol{n}) \cdot \boldsymbol{e}_{z}\right]\right|_{\gamma(t)} d z-\frac{1}{L} \int_{0}^{L}\left(\frac{d M}{d t} U+M \frac{d U}{d t}\right) d z . \quad \text { (Dynamic condition (z)) }
$$

The first term on the right hand side describes the viscous drag force (the jump across $\gamma(t)$ in the axial component of normal stress), the second term under the integral on the right hand-side describes the thrust generated by the displaced mass of the fluid $T=U d M / d t$, and the third term under the integral describes the "added mass effect", which is known to affect the total, "virtual mass" of a fluid-structure system by adding extra inertia to the system due to the displaced mass of the fluid surrounding the moving structure. Notice that since equation (38) is written in Eulerian coordinates, there is no Jacobian of the transformation from Eulerian to Lagrangian coordinates present here.

\subsection{The Reduced Model}

To derive the leading-order approximation of our fluid-structure interaction problem we begin, as usual, by first introducing the non-dimensional variables, which we denote by . The non-dimensional equations are obtained with the following scalings, where $\epsilon=R_{0} / L$ :

$$
z=L \tilde{z}, r=R_{0} \tilde{r}, u_{z}=V_{z} \tilde{u}_{z}, u_{r}=V_{r} \tilde{u}_{r}, p=\rho V_{z}^{2} \frac{1}{\epsilon^{2}} \tilde{p}, t=T \tilde{t}, \text { where } T=L / V_{z} .
$$

We plug these into the fluid and structure equations and ignore the terms of order $\epsilon^{2}$ or smaller to get the leading-order approximation of the problem. 
Fluid. We begin with the axial momentum equation (31). In non-dimensional variables the equation becomes:

$$
\begin{aligned}
\frac{\partial \tilde{u}_{z}}{\partial \tilde{t}}+\tilde{u}_{r} \frac{\partial \tilde{u}_{z}}{\partial \tilde{r}}+\tilde{u}_{z} \frac{\partial \tilde{u}_{z}}{\partial \tilde{z}}+\frac{1}{\epsilon^{2}} \frac{\partial \tilde{p}}{\partial \tilde{z}} & =\frac{\frac{\mu}{\rho} L}{V_{z} R_{0}^{2}}\left[\frac{1}{\tilde{r}} \frac{\partial}{\partial \tilde{r}}\left(\tilde{r} \frac{\partial \tilde{u}_{z}}{\partial \tilde{r}}\right)\right]=\frac{\frac{\mu}{\rho}}{L V_{z}} \frac{1}{\epsilon^{2}}\left[\frac{1}{\tilde{r}} \frac{\partial}{\partial \tilde{r}}\left(\tilde{r} \frac{\partial \tilde{u}_{z}}{\partial \tilde{r}}\right)\right] \\
& =\frac{1}{R e} \frac{1}{\epsilon^{2}}\left[\frac{1}{\tilde{r}} \frac{\partial}{\partial \tilde{r}}\left(\tilde{r} \frac{\partial \tilde{u}_{z}}{\partial \tilde{r}}\right)\right] .
\end{aligned}
$$

After multiplying the above equation by $\epsilon^{2}$ and ignoring the terms of order $\epsilon^{2}$, the leading-order equation that remains is

$$
\frac{\partial \tilde{p}}{\partial \tilde{z}}=\frac{1}{R e}\left[\frac{1}{\tilde{r}} \frac{\partial}{\partial \tilde{r}}\left(\tilde{r} \frac{\partial \tilde{u}_{z}}{\partial \tilde{r}}\right)\right] .
$$

After transforming this equation back into dimensional form we obtain the following leading-order balance of axial momentum:

$$
\frac{\partial p}{\partial z}=\mu\left[\frac{1}{r} \frac{\partial}{\partial r}\left(r \frac{\partial u_{z}}{\partial r}\right)\right] .
$$

We perform the same calculations for the balance of radial momentum equation (32), and the conservation of mass equation (33) and obtain the leading-order fluid equations, known as the lubrication model:

$$
\begin{aligned}
\frac{\partial p}{\partial z} & =\mu\left[\frac{1}{r} \frac{\partial}{\partial r}\left(r \frac{\partial u_{z}}{\partial r}\right)\right], \\
\frac{\partial p}{\partial r} & =0, \\
\frac{\partial}{\partial r}\left(r u_{r}\right)+\frac{\partial}{\partial z}\left(r u_{z}\right) & =0 .
\end{aligned}
$$

Structure. The elastodynamics of the structure is defined by equation (36), stated here one more time for convenience:

$$
m \frac{\partial^{2} R(z, t)}{\partial t^{2}}+\frac{4 E h}{3 R_{0}}\left(\frac{R(z, t)}{R_{0}}-1\right)=\left.\left[(\boldsymbol{\sigma} \boldsymbol{n}) \cdot \boldsymbol{e}_{r}\right]\right|_{\gamma(t)} J-\mathbf{F}^{e} \cdot \mathbf{e}_{r} .
$$

After taking the scalings (39) into account we obtain:

$$
\frac{\rho h R_{0}}{T^{2}} \frac{\partial^{2} \tilde{R}(z, t)}{\partial \tilde{t}^{2}}+\frac{4 E h}{3 R_{0}}(\tilde{R}(z, t)-1)=\left[-\rho V_{z}^{2} \frac{1}{\epsilon^{2}} \tilde{p}+2 \mu \frac{V_{r}}{R_{0}} \frac{\partial \tilde{u}_{r}}{\partial \tilde{r}}\right]_{\gamma(t)}-F_{r}^{e} \tilde{F}_{r}^{e}
$$

where $F_{r}^{e}$ is the average magnitude of external force applied to the surface of the swimmer to produce the traveling wave

$$
R(z, t)=H(z-s t)
$$

traveling with velocity $s<0$, where $H$ is a smooth approximation of the Heaviside function with

$$
R(0, t) \geq R(L, t) .
$$

We recall that $T=L / V_{z}$ and that $R_{0} / L=\epsilon$ and use this in the first term to obtain:

$$
\frac{m V_{z}^{2}}{L^{2}} \epsilon \frac{\partial^{2} \tilde{R}(z, t)}{\partial \tilde{t}^{2}}+\frac{4 E h}{3 R_{0}}(\tilde{R}(z, t)-1)=\left[-\rho V_{z}^{2} \frac{1}{\epsilon^{2}} \tilde{p}+2 \mu \frac{V_{r}}{R_{0}} \frac{\partial \tilde{u}_{r}}{\partial \tilde{r}}\right]_{\gamma(t)}-F_{r}^{e} \tilde{F}_{r}^{e}
$$

To continue, we need to specify the relative magnitude of the scaling parameters appearing in this equations. As we shall see below in the actual experiments with slender swimmers, the following holds true:

- $\frac{4 E h}{3 R_{0}}=\mathcal{O}\left(\rho V_{z}^{2} \frac{1}{\epsilon^{2}}\right)$, which is the scaling for the pressure. 
- $F_{r}^{e}<<\epsilon^{2}$.

Under these assumptions, after multiplying equation (42), the structure inertia term, the viscous component in the normal stress term, and the external forcing term drop out as they are of order $\epsilon^{2}$ or smaller, giving rise to the following, leading-order approximation of the dynamic coupling condition (36) in dimensional variables:

$$
-\left.[p]\right|_{\gamma(t)}=p-p_{\text {ext }}=\frac{4 E h}{3 R_{0}}\left(\frac{R(z, t)}{R_{0}}-1\right),
$$

where $p$ is the interior pressure exerted by the fluid on the cylinder wall.

The leading-order dimensional form of the kinematic coupling condition (continuity of velocity, i.e., no-slip) remains the same.

Leading-order approximation of the swimmer velocity $W(t)$. Velocity $W$ can be then calculated by integrating equation (38) with respect to $t$ to obtain

$$
m W(t)=-\frac{1}{L} \int_{0}^{L} M U d z+\left.\frac{1}{L} \int_{0}^{t} \int_{0}^{L}\left[(\boldsymbol{\sigma} \boldsymbol{n}) \cdot \boldsymbol{e}_{z}\right]\right|_{\Gamma(\tau)} d z d \tau .
$$

The flow rate $Q=M U$ can be easily calculated:

$$
Q(z, t)=\rho \pi R^{2}(z, t) U(z, t),
$$

where $U$ is the cross-sectional average of the axial component of fluid velocity, given by (37). Therefore, we now have

$$
m W(t)=-\rho \frac{1}{L} \int_{0}^{L} \pi R^{2}(z, t) U(z, t) d z+\left.\frac{1}{L} \int_{0}^{t} \int_{0}^{L}\left[(\boldsymbol{\sigma n}) \cdot \boldsymbol{e}_{z}\right]\right|_{\Gamma(\tau)} d z d \tau .
$$

The expression for the viscous drag force term is obtained from the definition of the Cauchy stress tensor $\boldsymbol{\sigma}$ given in (34), and by taking into account $\boldsymbol{n} \approx \boldsymbol{e}_{r}$, to obtain

$$
\left.\left[(\boldsymbol{\sigma} \boldsymbol{n}) \cdot \boldsymbol{e}_{z}\right]\right|_{\gamma(t)}=\left.\left[\mu\left(\frac{\partial u_{r}}{\partial z}+\frac{\partial u_{z}}{\partial r}\right)\right]\right|_{\gamma(t)} .
$$

As before, the square brackets denote the jump in the stress across $\gamma(t)$. We calculate the relative sizes of the terms in the viscous drag force by using the non-dimensional variables. Within the cylinder we have

$$
\frac{u_{r}}{L}=\epsilon^{2} \frac{V_{z}}{R_{0}},
$$

and so we can ignore the term with $\partial u_{r} / \partial z$. Outside the swimmer, we assume that the radial component of the velocity is much smaller than the axial component, since the fluid is initially at rest, and is perturbed only locally due to the motion of the swimmer in the axial direction. Thus, the leading-order equation for the swimmer velocity becomes

$$
m W(t)=-\rho \frac{1}{L} \int_{0}^{L} \pi R^{2}(z, t) U(z, t) d z+\left.\mu \frac{1}{L} \int_{0}^{t} \int_{0}^{L}\left[\frac{\partial u_{z}}{\partial r}\right]\right|_{\Gamma(\tau)} d z d \tau .
$$

Thus, the velocity of the swimmer at time $t$ is affected by the current propulsion by the fluid within the swimmer (i.e., the momentum of the fluid created within the swimmer due to the peristaltic motion), and by the (time-) history of the viscous drag force exerted onto the swimmer until time $t$.

Explicit calculation of the swimmer velocity $W(t)$. We conclude this section by using the fluid and structure equations to explicitly calculate the quantities in the expression for the swimmer velocity $W(t)$ given above. 
1. The viscous drag force: We will be able to explicitly calculate the viscous drag force by calculating the axial component of velocity $u_{z}$ from the fluid flow equations. By integrating the leading order conservation of axial momentum equation $(41)$ over $(0, r)$ we obtain:

$$
\frac{\partial u_{z}}{\partial r}=\frac{r}{2 \mu} \frac{\partial p}{\partial z}
$$

Integrating this equation over $(r, R)$, and after taking into account the no-slip condition at $r=R$ one obtains:

$$
u_{z}=\frac{1}{4 \mu}\left(r^{2}-R^{2}\right) \frac{\partial p}{\partial z}
$$

which is the Poiseuille velocity profile inside the cylinder. Outside the cylinder we will be assuming that the axial velocity profile is Poiseuille-like as shown in Figure 9, with $\partial u_{z} / \partial r=0$ on the outside of $\gamma(t)$. With this, the jump in the viscous drag force becomes

$$
\left.\left[\frac{\partial u_{z}}{\partial r}\right]\right|_{\Gamma(t)}=-\frac{R(z, t)}{2 \mu} \frac{\partial p}{\partial z}
$$

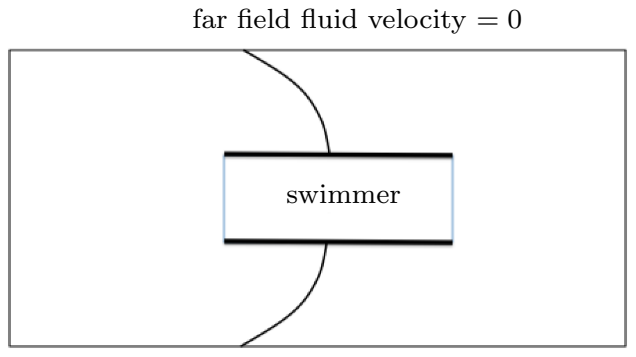

Figure 9: Assumption on the velocity profile external to the swimmer, generated by the swimmer moving in low Reynolds number viscous fluid with no-slip condition.

2. The cross-sectional velocity: Now, we can also easily calculate the cross-sectional average of $u_{z}$ by using (47) to obtain:

$$
\begin{aligned}
U(z, t) & =\frac{2}{R^{2}} \int_{0}^{R} u_{z} r d r=\frac{1}{2 \mu R^{2}} \int_{0}^{R}\left(r^{2}-R^{2}\right) \frac{\partial p}{\partial z} r d r=\frac{1}{2 \mu R^{2}} \frac{\partial p}{\partial z}\left(\frac{R^{4}}{4}-\frac{R^{4}}{2}\right) \\
& =-\frac{1}{8 \mu} \frac{\partial p}{\partial z} R^{2}(z, t) .
\end{aligned}
$$

3. The mass flux: From here, the mass flux (flow rate) $Q$ is equal to:

$$
Q(z, t)=\rho \pi R^{2}(z, t) U(z, t)=-\frac{\pi \rho}{8 \mu} \frac{\partial p}{\partial z} R^{4}(z, t) .
$$

Thus, the flow rate changes with the 4 th power of the radius.

Now, equation (4.2) for the calculation of the swimmer's velocity $W$ becomes

$$
m W(t)=\frac{\pi \rho}{8 \mu L} \int_{0}^{L} \frac{\partial p}{\partial z}(z, t) R^{4}(z, t) d z-\frac{1}{2 L} \int_{0}^{t} \int_{0}^{L} \frac{\partial p}{\partial z}(z, \tau) R(z, \tau) d z d \tau .
$$

We can now use the leading order pressure-radius relationship given by (43) to express the pressure gradient above in terms of the change in the radius. Assuming that the pressure exterior to the swimmer is approximately constant, we obtain

$$
\frac{\partial p}{\partial z}=\frac{4 E h}{3 R_{0}^{2}} \frac{\partial R}{\partial z} .
$$

From here, finally, we get the equation for the swimmer velocity all in terms of $R$ as follows: 


$$
m W(t)=\frac{2 E h}{3 L R_{0}^{2}}\left\{\frac{\pi \rho}{4 \mu} \int_{0}^{L} \frac{\partial R}{\partial z}(z, t) R^{4}(z, t) d z-\int_{0}^{t} \int_{0}^{L} \frac{\partial R}{\partial z}(z, \tau) R(z, \tau) d z d \tau\right\}
$$

This can be further simplified to

$$
m W(t)=\frac{2 E h}{3 L R_{0}^{2}}\left\{\frac{\pi \rho}{20 \mu} \int_{0}^{L} \frac{\partial R^{5}}{\partial z}(z, t) d z-\frac{1}{2} \int_{0}^{t} \int_{0}^{L} \frac{\partial R^{2}}{\partial z}(z, \tau) d z d \tau\right\} .
$$

After integrating the right hand-side with respect to $z$ we obtain:

$$
m W(t)=\frac{2 E h}{3 L R_{0}^{2}}\left\{\frac{\pi \rho}{4 \mu}\left(\frac{R^{5}(L, t)}{5}-\frac{R^{5}(0, t)}{5}\right)-\int_{0}^{t}\left(\frac{R^{2}(L, \tau)}{2}-\frac{R^{2}(0, \tau)}{2}\right) d \tau\right\}
$$

Result (52) indicates that the speed of the swimmer does not depend on the precise form of the peristaltic wave (i.e., pulses or constrictions), which was also observed in [2] for a different but related problem of a spherical swimmer propelled by a peristaltic motion of a channel internal to the spherical swimmer with a rigid spherical outer boundary.

Since $R$ is small $R<<1$, we see that the dominant term is the term containing $R^{2}$, which is the viscous drag force term. Furthermore, in the peristaltic wave $H(z-s t)$ with $R(0, t) \geq R(L, t)$ as mentioned earlier, the difference $R^{2}(L, t)-R^{2}(0, t)$ is negative, and so the sign of this term is positive. Notice that this term accounts for the "displaced mass" through the change in the (scaled) cross-sectional area of the cylinder which is captured by the term $R^{2}(L, t)-R^{2}(0, t)$. Thus, the jump across $\gamma(t)$ in the viscous drag force is proportional to the displaced fluid mass by the swimmer.

We can simplify this expression even further by defining our traveling wave as

$$
R(z, t)=H(z-s t)= \begin{cases}R_{M A X}, & z \in\left[0, z^{*}(t)\right) \\ R_{M I N}, & z \in\left(z^{*}(t), L\right]\end{cases}
$$

where

$$
z^{*}(t)=L-s t .
$$

The wave reaches the left end of the swimmer at time $T=L / s$. Therefore, we obtain that for $t \in(0, T]$,

$$
W(t)=\frac{2 E h}{3 m L R_{0}^{2}}\left\{\frac{\pi \rho}{4 \mu}\left(\frac{R_{M I N}^{5}}{5}-\frac{R_{M A X}^{5}}{5}\right)-\left(\frac{R_{M I N}^{2}}{2}-\frac{R_{M A X}^{2}}{2}\right) t\right\}
$$

The leading-order approximation is given by:

$$
W(t) \approx \frac{2 E h}{3 m L R_{0}^{2}}\left(R_{M A X}^{2}-R_{M I N}^{2}\right) t,
$$

and for $t=T$, we have

$$
W(T) \approx \frac{2 E h}{3 m L R_{0}^{2}}\left(R_{M A X}^{2}-R_{M I N}^{2}\right) \frac{L}{2 s}=\frac{2 E h}{6 m s R_{0}^{2}}\left(R_{M A X}^{2}-R_{M I N}^{2}\right) .
$$

\subsection{Validation}

To validate the model we used the 3D computer simulations described in the first part of the paper to compare the velocity of a slender swimmer obtained using 3D simulations with those given by the 1D model (54) or (52) above. We considered a slender swimmer with

$$
R_{0}=0.075 \mu m, L=0.667 \mu m, \epsilon=0.1124 .
$$

In order to be able to use the formulas (54) or (52) we had to estimate the Youngs modulus of the overall structure. Recall that we have the elasticity constants for each of the springs that are modeling 

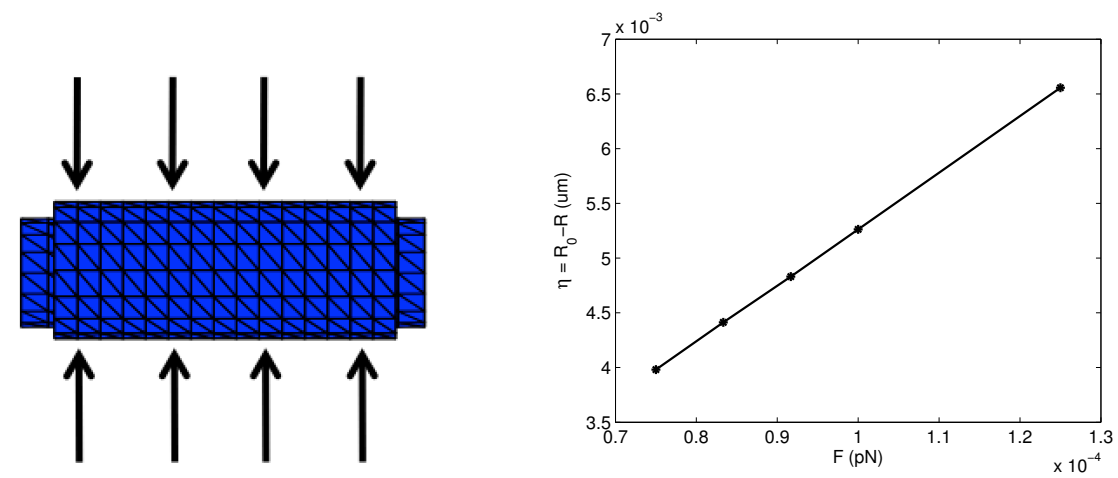

Figure 10: Left: A sketch of how normal force was applied to deform the swimmer to a uniform radius. Right: Calculated relationship between force and displacement, determining the constant $C_{1}=4 E h / 3 R_{0}^{2}=$ Force/Displacement. We see that the relationship is very close to linear for these displacements.

the cylindrical membrane, but not the overall Youngs modulus of the cylinder. For this purpose we calculated the constant

$$
C_{1}=4 E h /\left(3 R_{0}^{2}\right),
$$

which appears as the proportionality constant in the pressure-radius relationship (50) governing the elastic structure problem (30). We applied a series of different (pressure) loadings displacing the elastic cylinder by a certain (uniform) radius, as shown in Figure 10 (left), to obtain the following values for $C_{1}$ :

\begin{tabular}{|l|c|c|c|c|c|}
\hline$F(p N)$ & $7.5 \mathrm{e}-005$ & $8.333 \mathrm{e}-005$ & $9.166 \mathrm{e}-005$ & $1.0 \mathrm{e}-004$ & $1.24 \mathrm{e}-004$ \\
\hline$R_{0}-R(\mu m)$ & 0.003981 & 0.004412 & 0.004831 & 0.005262 & 0.006556 \\
\hline$C_{1}=F /\left(R_{0}-R\right)\left(\mu g /(m s)^{2}\right)$ & $1.8839 \mathrm{e}-005$ & $1.8917 \mathrm{e}-005$ & $1.90 \mathrm{e}-005$ & $1.90 \mathrm{e}-005$ & $1.9083 \mathrm{e}-005$ \\
\hline
\end{tabular}

We conclude that for these displacements, the pressure-radius relationship is very close to linear, indeed, see Figure 10 (right), and that the proportionality constant $C_{1}$ is well approximated by

$$
C_{1}=1.90 \times 10^{-5} \frac{\mu g}{(m s)^{2}} .
$$

The remaining parameters in the problem are the following: $\rho=10^{-6} \mu \mathrm{g} /(\mu \mathrm{m})^{3}, \mu=1.2 \times 10^{-3} \mu \mathrm{g} /(\mu \mathrm{m}$. $m s$ ), and $m=7.92 \times 10^{-4} \mu \mathrm{g} / \mu \mathrm{m}$.

Figure 11 shows a comparison between the numerically simulated velocity of the swimmer described above (red curve), and the velocity of the swimmer obtained using the 1D model (54). We plotted the solution to the 1D model only from the point from where the velocity of the swimmer becomes positive. We see excellent agreement between the two.

\subsection{Predictions of the $1 D$ model for the examples studied in Sections 3.1 and 3.2.}

We used the 1D model presented above to see how well can the model predict the distance traveled by the swimmer for the different cases studied in Sections 3.1 and 3.2. Recall, in Section 3.1 the influence of the ratio between the swimmer reference radius $R_{0}$ and length $L$ on the distance traveled by the swimmer was studied, and in Section 3.2 the influence of the deformed radius relative to the reference radius $R_{0}$ on the distance traveled by the swimmer was studied. In those examples the swimmers were not necessary slender, and the deformation of the swimmer was not necessarily small, so we expect some discrepancies between the 1D and 3D model for the parameter values outside of the validity of the 1D model. We used the 1D model (54) with mass density per unit length of $2.25 \times 10^{-3} \mu \mathrm{g} / \mu \mathrm{m}$ to calculate 


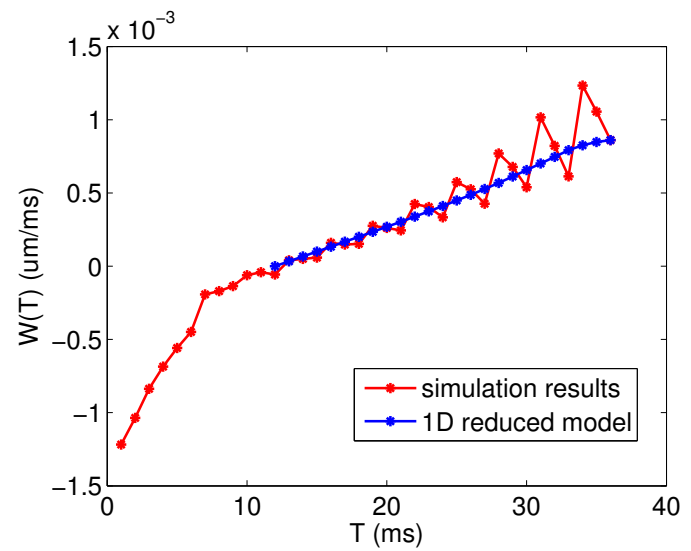

Figure 11: A comparison between swimmer velocity obtained using full 3D simulations (red) and the reduced 1D model (54) (blue). We see excellent agreement between the two in the regime where the swimmer velocity is positive.

the total distance traveled by the swimmer at time $T$ when the traveling wave reaches the left end of the swimmer:

$$
X(T)=W(T) T .
$$

We compared predictions provided by the 1D model with the 3D simulation results. Figure 12 (left) shows a comparison for the cases studied in Section 3.1, and Figure 12 (right) shows a comparison for the cases studied in Section 3.2. We see that there is excellent agreement between the 1D model and
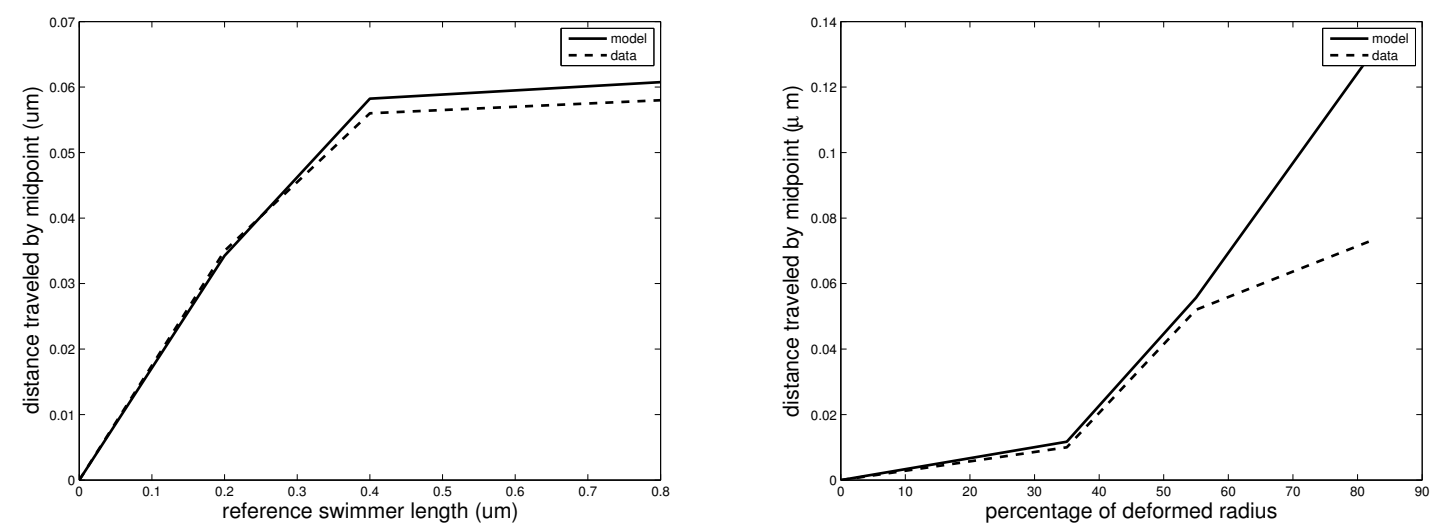

Figure 12: Comparison between the 1D model and 3D simulations for the distance traveled by the swimmers studied in Sections 3.1 (left) and 3.2 (right).

3D simulations, especially for the cases when the swimmer displacement is less than or equal to $50 \%$ of the reference radius. We see in Figure 12 (right) that for larger displacements the 1D model, which assumes linear elasticity, over estimates the distance traveled by the swimmer because for such large displacements the nonlinear structure effects in the full 3D model become much more prominent.

We conclude that for small-to-medium displacements the 1D model captures well the dependence of swimmer velocity on the parameters in the problem.

\subsection{Multiple contractions of the swimmers}

We conclude this manuscript by investigating the influence of multiple swimmer contractions, i.e., multiple cycles of force application on the swimmer, on the distance traveled by the swimmer over some 
given, fixed time interval. We have some interesting results to report.

From the previous simulations and the results presented above, one can see that while larger deformations of the swimmer, which generate larger fluid mass displacements, imply farther distances traveled by the swimmer, the time it takes the swimmer to deform under the action of a given (fixed) force, and the time it takes the swimmer to re-assume its original equilibrium state before the next peristaltic wave can be applied, is longer in those cases. This is due to friction, i.e., viscous effects exerted by the fluid onto the swimmer, and due to the "added mass effect" of the surrounding fluid. Namely, the viscosity of the surrounding fluid makes elastic swimmers behave as a (damped) viscoelastic structure, reacting to a given application of force and to the release of force with a delay. The added mass effect, or virtual mass, is the inertia added to a system because the swimmer needs to displace a certain volume of fluid of density $\rho$. The larger the displaced volume and fluid density, the larger the added mass effect. Also, the larger the the difference in acceleration between the fluid and the swimmer, the larger the added mass effect. When density of the structure is much bigger than that of the fluid (e.g. in aeroelasticity) the added mass effect is small relative the structure inertia. However, in our study this is not the case since the elastic swimmer and the fluid surrounding the swimmer have comparable densities, giving rise to a strong added mass effect. This is why we see that it takes longer for the swimmers producing larger fluid mass displacements shown in Figures 6 and 7 in red, to re-assume their original, equilibrium state. These effects influence the distance traveled by the swimmer over a fixed amount of time for multiple applications of force generating several peristaltic waves in the swimmer membrane.
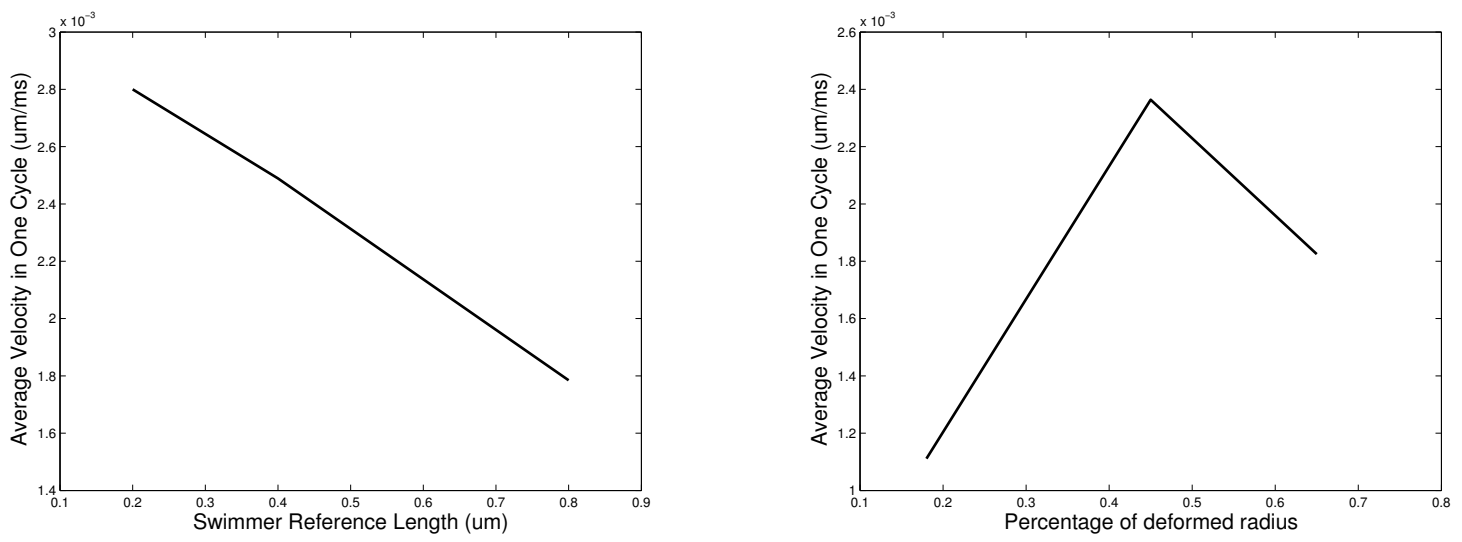

Figure 13: Average velocity of swimmers during one cycle of force application. Left: Swimmers with different lengths $(L=0.2,0.4,0.8 \mu \mathrm{m})$ and same reference radius $R_{0}=0.125 \mu \mathrm{m}$. Right: Swimmers with same reference length $(L=0.4 \mu \mathrm{m})$ and radius $\left(R_{0}=0.125 \mu \mathrm{m}\right)$ but different traveling wave amplitude (percentage of deformed radius $0.18 \%, 0.45 \%, 0.65 \%$ ).

Additionally, we calculated from 3D simulations the average velocity of each swimmer in one cycle and obtained interesting results, shown in Figure 13. The picture on the left in Figure 13 shows the average swimmer velocity for the swimmers with different aspect ratio $\epsilon$, studied in Section 3.1. We see that the shorter the swimmer, i.e., the larger the aspect ratio, the larger the average velocity of the swimmer. The picture on the right shows the average swimmer velocity for the swimmers with different relative amplitude $\lambda$ of the traveling wave, studied in Section 3.2. We can see that the largest average velocity is associated with the swimmers in the roughly $50 \%$ deformation range, i.e., the deformed radius being roughly $50 \%$ of the reference radius.

All these factors will influence the distance traveled by the swimmers within a fixed time interval, after multiple cycles of application of force on the swimmer membrane. For each swimmer the force, described in Section 3, was applied to produce one peristaltic wave traversing the swimmer from right to left. A restoring force was then applied in the opposite direction, as described in Section 3, to recover the 

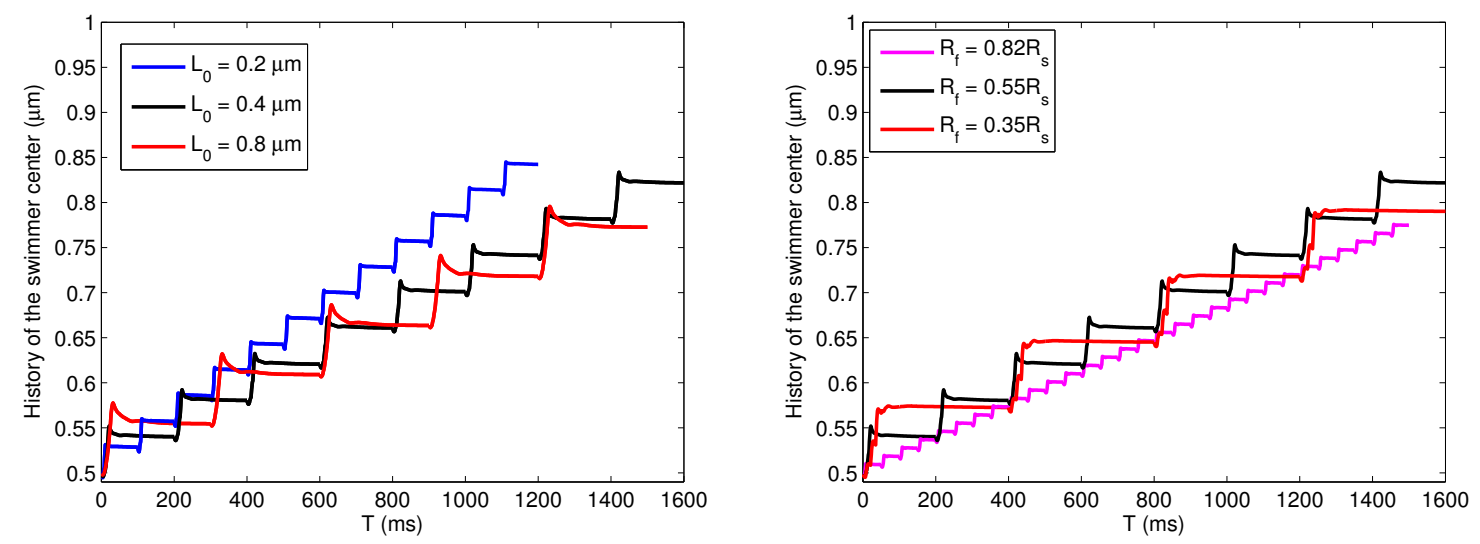

Figure 14: The figures show history of swimmers center for multiple cycles of force application. Left: comparison between swimmers of different reference length, same reference radius $R_{0}=0.125 \mu \mathrm{m}$, and same deformed radius $R_{f}=0.55 R_{0}$, c.f. Figure 6. Right: comparison between swimmers with different deformed radius, same reference radius $R_{0}=0.125 \mu \mathrm{m}$ and same reference length $L=0.4 \mu m$, c.f. Figure 8 .

reference configuration of the swimmer. The total energy was checked to see if it was close to zero within some $\delta$ tolerance, indicating that the reference, equilibrium state of the swimmer had been achieved. As soon as this condition was satisfied, another cycle of force was applied to produce the second perstaltic wave, and so on. The same force density $F_{\text {ext }} / N_{n}$ was applied in all cases. Figure 14 (left) shows the swimmers, discussed in Section 3.1, exposed to multiple cycles of force for the duration of $1.2 \mathrm{~s}$. Recall that all the swimmers in this case had the same reference radius $R_{0}$, and the same deformed radius $R_{f}$. The only difference was the reference length in each swimmer. Figure 14 (right) shows the swimmers, discussed in Section 3.2, exposed to multiple cycles of force for the duration of $1.5 \mathrm{~s}$.

Conclusions. We observe in Figure 14 (left) that the swimmer that moved the farthest during the time interval $(0,1.2) \mathrm{s}$ was the swimmer with the shortest reference length $L_{0}=0.2 \mu \mathrm{m}$, i.e., largest aspect ratio $\epsilon=R_{0} / L$. Even though the displaced mass during one cycle was the smallest for this swimmer, the rate of displaced mass per second is the largest, as shown in Figure 13 (left). This, combined with the smallest added mass effect associated with this swimmer, produces the fastest swimmer overall when swimmers with different aspect ratio are considered.

Figure 14 (right) shows, on the other hand, that all three swimmers produce roughly the same distance traveled during the time interval $(0,1.5) s$. One can notice that the average slope corresponding to the black curve is slightly bigger, indicating that for longer distances the motion of the swimmer with the deformed swimmer radius of roughly $50 \%$ of the reference radius, is the "optimal" solution. This is consistent with the result shown in Figure 13 (right), which indicates that swimmers with roughly $50 \%$ of deformation have the largest average velocity in one cycle of force application.

Thus, our study indicates that fast swimmers are those with large aspect ratios whose radius deforms roughly $50 \%$ in each cycle. Such swimmers are capable of generating a large number of contractions per unit time because of their short length, producing the largest rate of change of displaced mass per unit time.

\section{Conclusions}

We conclude this manuscript by showing in Figure 15 three "representative" snap shots of the motion of our "optimal swimmer" with $R_{0}=0.125, L=0.2$ and $R_{f}=0.55 R_{0}$ over multiple (10) cycles, corresponding to the blue curve in Figure 14 (left). We calculated the distance traveled by the swimmer 


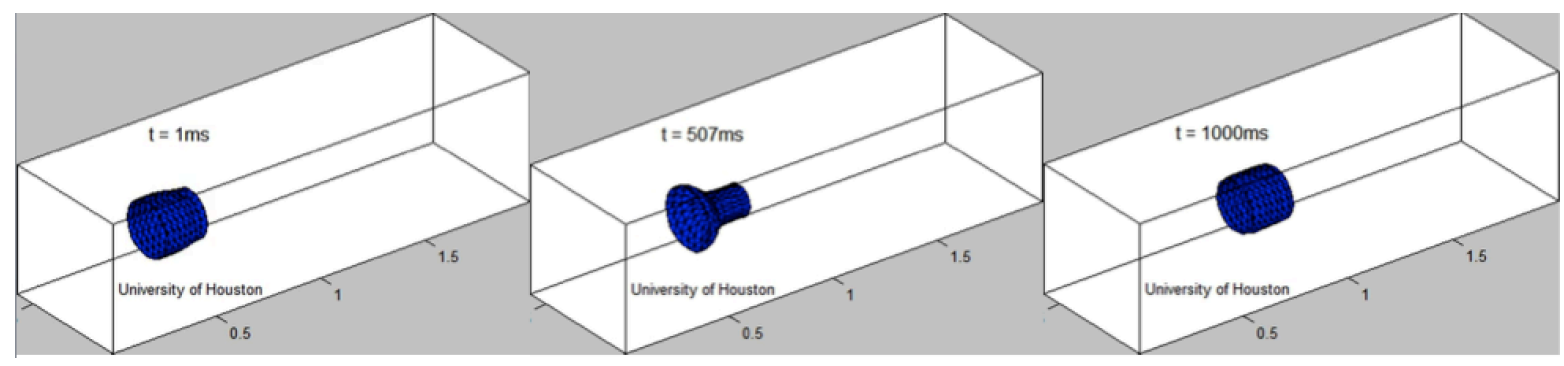

Figure 15: Motion of the swimmer with $R_{0}=0.125, L=0.2$ and $R_{f}=0.55 R_{0}$ over multiple (10) cycles, corresponding to the blue curve in Figure 14 (left). Three "representative" snap shots are shown at $t=1 \mathrm{~ms}, t=507 \mathrm{~ms}$, and $t=1000 \mathrm{~ms}$.

and obtained that within one second our "optimal swimmer" traversed the distance equal to 1.5 of the swimmer lengths. Figure 16 shows the first and last snap shot superimposed, indicating the traversed distance.

The results of our work are consistent with the results reported in the review article [11] published in 2014 in Micromachines. In fact, our swimmers are at the top of the class of all artificial swimmers swimming at low Reynolds numbers reported in [11]. More precisely, the authors of [11] obtained a diagram comparing propulsion speed versus Reynolds numbers for 18 artificial (manufactured) and natural micro-swimmers. It was concluded that artificial micro-swimmers in general travel slower than the natural ones, and that at low Reynolds numbers the speed of artificial micro-swimmers is between 0.6 and 2 body length per second at the Reynolds numbers around $10^{-6}$. Therefore, the results of our research are very relevant, and are a first step in our long-term goal to design a medical micro-swimmer based on the contractions of a cylindrical membrane lined with cardiac myocytes capable of producing coordinated contractions [15].

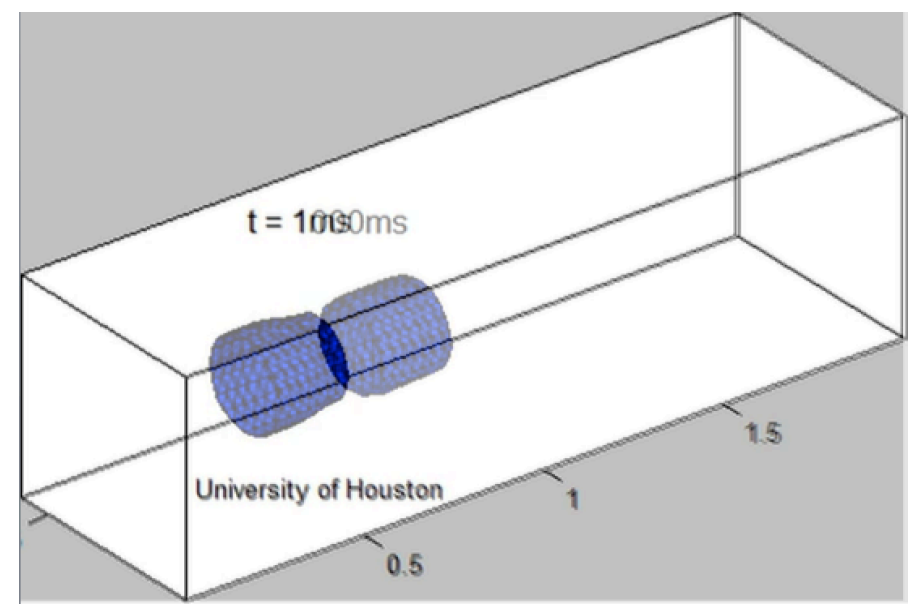

Figure 16: Two snap shots from Figure 15 (first and last) superimposed. The figure indicates that the swimmer has traversed the distance equal to 1.5 reference lengths.

\section{Acknowledgments}

The authors would like to thank Dr. Richard D. James for bringing this problem to their attention while meeting at Oxford University in 2014. The research in this work has been partially supported by the National Science Foundation under grants NSF DMS-1262385 (Čanić, Quaini, Shi), NSF DMS1311709 (Čanić), NSF DMS-1318763 (Čanić), and NSF/NIGMS DMS-1263572 (Čanić, Quaini, Shi), 
NSF DMS-1418308 (Pan).

\section{References}

[1] J. Adams, P. Swarztrauber, and R. Sweet, FISHPAK: A package of Fortran subprograms for the solution of separable elliptic partial differential equations, The National Center for Atmospheric Research, Boulder, CO, 1980.

[2] A. Adjari, and H. A. Stone, A note on swimming using internally generated traveling waves, Physics of Fluids, 11(5), 1999, 1275-1277.

[3] W. Alt, and M. Dembo, Cytoplasm dynamics and cell motion: twophase flow models, Mathematical Biosciences, 156, 1999, 207-228.

[4] V. Aranda, R. Cortez and L. Fauci. A model of Stokesian peristalsis and vesicle transport in a three-dimensional closed cavity. J. Biomechanics, Vol. 48 (9), (2015) 1631-8.

[5] C.J. Brokaw, Simulating the effects of fluid viscosity on the behavior of sperm flagella, Mathematical Methods in the Applied Sciences, 24, 2001, 1351-1365.

[6] S. Čanić, E-H. Kim. Mathematical Analysis of the Quasilinear Effects in a Hyperbolic Model of Blood Flow through Compliant Axisymmetric Vessels, Mathematical Methods in Applied Sciences, 26 (14), 2003, 1161-1186.

[7] E.J. Dean, and R. Glowinski, A wave equation approach to the numerical solution of the NavierStokes equations for incompressible viscous flow, C.R. Acad. Sc. Paris, Série 1, 325, 1997, 783791.

[8] E.J. Dean, R. Glowinski, and T.W. Pan, A wave equation approach to the numerical simulation of incompressible viscous fluid flow modeled by the Navier-Stokes equations, In Mathematical and Numerical Aspects of Wave Propagation, De Santo JA (Ed.), SIAM: Philadelphia, 1998, 65-74.

[9] R. Dreyfus, J. Baudry, M.L. Roper, M. Fermigier, H.A. Stone, and J. Bibette, Microscopic artificial swimmers, Nature, 437, 2005, 862-865.

[10] T. Ebefors, and G. Stemme, The MEMS handbook, Chapter 28- Micro-robotics, CRC Press, 2002, $28-1-28-42$.

[11] J. Feng, and S. K. Cho, Mini and Micro Propulsion for Medical Swimmers (Review), Micromachines $5,2014,97-113$.

[12] R. Glowinski, Finite element methods for incompressible viscous flow, In Handbook of Numerical Analysis, Vol. IX, Ciarlet PG and Lions JL (Eds.), North-Holland: Amsterdam, 2003 (Chapters II, V, VI, VII, VIII).

[13] S. Guo, Y. Okuda, and K. Asaka, Hybrid type of underwater microbiped robot with walking and swimming motions, IEEE Conference on Robotics and Automation (ICRA'05), 3, 2004, 16041609 .

[14] C. Hamlet, L. Fauci and E.D. Tytell. The effect of intrinsic muscular nonlinearities on the energetics of locomotion in a computational model of an anguilliform swimmer. J. Theor. Biol. To appear (2015).

[15] L. Khait, and R.K. Birla, Cell-based cardiac pumps and tissue-engineered ventricles, Regenerative Medicine 2(4), 2007, 391-406 
[16] G. Kósa, M. Shoham, and M. Zaaroor, Propulsion Method for Swimming Micro Robots, IEEE Transaction on Robotics, 23, 2007, 137-150.

[17] G. Kósa, and Péter Jakab, Nobuhiko Hata, Ferenc Jólesz, Zipi Neubach, Moshe Shoham, Menashe Zaaroor, and Gábor Székely, Flagellar swimming for medical micro robots: Theory, experiments and application, Biomedical Robotics and Biomechatronics, 2008. BioRob 2008. 2nd IEEE RAS \& EMBS International Conference 2008, 258 - 263.

[18] M.A. Mohamed, M.K. Hogan, N.M. Patel, Z-w. Tao, L. Gutierrez, and R.K. Birla, Establishing the Framework for Tissue Engineered Heart Pumps, Cardiovacular Engineering and Technology, Published online January 2015.

[19] Y. Okada, S. Takeda, Y. Tanaka, J.C. Belmonte, and N. Hirokawa, Mechanism of Nodal Flow: A Conserved Symmetry Breaking Event in Left-Right Axis Determination, Cell, 121, 2005, 633-644.

[20] T.W. Pan, and R. Glowinski, Direct simulation of the motion of neutrally buoyant balls in a three-dimensional Poiseuille flow, C. R. Mecanique, 333, 2005, 884-895.

[21] C.S. Peskin, Numerical analysis of blood flow in the heart, J. Comput. Phys., 25, 1977, 220-252.

[22] C.S. Peskin, and McQueen D.M., Modeling prosthetic heart valves for numerical analysis of blood flow in the heart, J. Comput. Phys., 37, 1980, 11332.

[23] C.S. Peskin, The immersed boundary method, Acta Numer., 11, 2002, 479-517.

[24] J. Simons, L. Fauci and R. Cortez. A fully three-dimensional model of the interaction of driven elastic filaments in a Stokes flow with applications to sperm motility. J. Biomechanics, 48 (9), 2015, 1639-1651.

[25] L. Shi, T.W. Pan, and R. Glowinski, Three-dimensional simulation of red blood cell motion in Poiseuille flows, International Journal for Numerical Methods in Fluids, 76, 2014, 397-415.

[26] D. Takagi, and N. J. Balmforth, Peristaltic pumping of rigid objects in an elastic tube, J. Fluid Mech., 672, 2011, 219-244.

[27] M. Tanaka, S. Wada and M. Nakamura. Computational Biomechanics: Theoretical Background and Biological/Biomedical Problems. Springer, 2012 ISBN-13: 978-4431540724.

[28] E. Tytell, C. Hsu, T. Williams, A. Cohen and L. Fauci. Interactions between internal forces, body stiffness, and fluid environment in a neuromechanical model of lamprey swimming. Proc. National Acad. of Sciences 107, 2010, 19832-19837.

[29] S. Wada, and R. Kobayashi, Numerical simulation of various shape changes of a swollen red blood cell by decrease of its volume, Trans. JSME. Ser. A, 69, 2003, 14-21.

[30] T.Y.T. Wu, C.J. Brokaw, and C. Bremmen Edt, Swimming and flying in nature, New York: Plenum Press, 1975, pp.161-173.

[31] K.B. Yesin, K. Vollmers, and B.J. Nelson, Modeling and control of untethered biomicrorobots in a fluidic environment using electromagnetic fields, International Journal of Robotics Research, 25, 2006, 527-536. 